NASA/TM-2001-210391

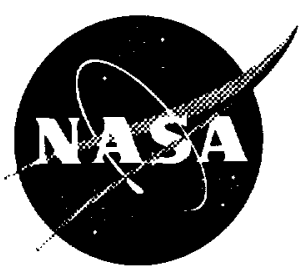

\title{
Nonstationary Dynamics Data Analysis With Wavelet-SVD Filtering
}

Marty Brenner

NASA Dryden Flight Research Center

Edwards, California

Dale Groutage

Naval Surface Warfare Center (NSWC)

Bremerton, Washington 


\section{The NASA STI Program Office...in Profile}

Since its founding, NASA has been dedicated to the advancement of aeronautics and space science. The NASA Scientific and Technical Information (STI) Program Office plays a key part in helping NASA maintain this important role.

The NASA STI Program Office is operated by Langley Research Center, the lead center for NASA's scientific and technical information. The NASA STI Program Office provides access to the NASA STI Database, the largest collection of aeronautical and space science STI in the world. The Program Office is also NASA's institutional mechanism for disseminating the results of its research and development activities. These results are published by NASA in the NASA STI Report Series, which includes the following report types:

- TECHNICAL PUBLICATION. Reports of completed research or a major significant phase of research that present the results of NASA programs and include extensive data or theoretical analysis. Includes compilations of significant scientific and technical data and information deemed to be of continuing reference value. NASA's counterpart of peer-reviewed formal professional papers but has less stringent limitations on manuscript length and extent of graphic presentations.

- TECHNICAL MEMORANDUM. Scientific and technical findings that are preliminary or of specialized interest, e.g., quick release reports, working papers, and bibliographies that contain minimal annotation. Does not contain extensive analysis.

- CONTRACTOR REPORT. Scientific and technical findings by NASA-sponsored contractors and grantees.
- CONFERENCE PUBLICATION. Collected papers from scientific and technical conferences, symposia, seminars, or other meetings sponsored or cosponsored by NASA.

- SPECIAL PUBLICATION. Scientific, technical, or historical information from NASA programs, projects, and mission, often concerned with subjects having substantial public interest.

- TECHNICAL TRANSLATION. Englishlanguage translations of foreign scientific and technical material pertinent to NASA's mission.

Specialized services that complement the STI Program Office's diverse offerings include creating custom thesauri, building customized databases, organizing and publishing research results...even providing videos.

For more information about the NASA STI Program Office, see the following:

- Access the NASA STI Program Home Page at http://www.sti.nasa.gov

- E-mail your question via the Internet to help@sti.nasa.gov

- Fax your question to the NASA Access Help Desk at (301) 621-0134

- Telephone the NASA Access Help Desk at (301) 621-0390

- Write to: NASA Access Help Desk NASA Center for AeroSpace Information 7121 Standard Drive Hanover, MD 21076-1320 


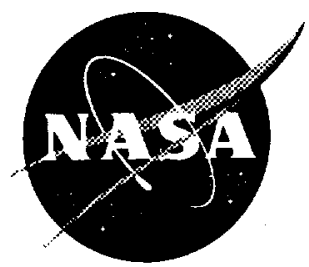

\section{Nonstationary Dynamics Data Analysis With Wavelet-SVD Filtering}

Marty Brenner

NASA Dryden Flight Research Center

Edwards, California

Dale Groutage

Naval Surface Warfare Center (NSWC)

Bremerton, Washington

National Aeronautics and

Space Administration

Dryden Flight Research Center

Edwards, California 93523-0273

April 2001 


\section{NOTICE}

Use of trade names or names of manufacturers in this document does not constitute an official endorsement of such products or manufacturers, either expressed or implied, by the National Aeronautics and Space Administration.

Available from the following:

NASA Center for AeroSpace Information (CASI) 7121 Standard Drive

Hanover, MD 21076-1320

(301) 621-0390
National Technical Information Service (NTIS) 5285 Port Royal Road Springfield, VA 22161-2171 (703) $487-4650$ 


\section{NONSTATIONARY DYNAMICS DATA ANALYSIS WITH WAVELET-SVD FILTERING}

\author{
Marty Brenner* \\ Aerostructures Branch \\ NASA Dryden Flight Research Center \\ Edwards, CA 93523-0273
}

\author{
Dale Groutage ${ }^{\dagger}$ \\ Puget Sound Detachment \\ Naval Surface Warfare Center (NSWC) \\ Bremerton, WA 98314-5215
}

\begin{abstract}
Nonstationary time-frequency analysis is used for identification and classification of aeroelastic and aeroservoelastic dynamics. Time-frequency multiscale wavelet processing generates discrete energy density distributions. The distributions are processed using the singular value decomposition (SVD). Discrete density functions derived from the SVD generate moments that detect the principal features in the data. The SVD standard basis vectors are applied and then compared with a transformed-SVD, or TSVD, which reduces the number of features into more compact energy density concentrations. Finally, from the feature extraction, wavelet-based modal parameter estimation is applied.

The primary objective is the automation of time-frequency analysis with modal system identification. The contribution is a more general approach in which distinct analysis tools are merged into a unified procedure for linear and nonlinear data analysis. This method is first applied to aeroelastic pitchplunge wing section models. Instability is detected in the linear system, and nonlinear dynamics are observed from the time-frequency map and parameter estimates of the nonlinear system. Aeroelastic and aeroservoelastic flight data from the DAST (drone for aerodynamic and structural testing) and F18 aircraft are also investigated and comparisons made between the SVD and TSVD results. Input-output data is used to show that this process is an efficient and reliable tool for automated on-line analysis.
\end{abstract}

\section{Nomenclature}

$a$

DAST

wavelet scale

DWT drone for aerodynamic and structural testing discrete wavelet transform

805.276.3793, marty.brenner@dfrc.nasa.gov

$\dagger$ 360.476.5927, groutagefd@nswccd.navy.mil

Copyright (C) 2001 by the American Institute of Aeronautics and Astronautics, Inc. No copyright is asserted in the United States under Title 17 , U.S. Code. The U.S. Government has a royalty-free license to exercise all rights under the copyright claimed herein for government purposes. All other rights are reserved by the copyright owner.

$\begin{array}{ll}F_{i} & \text { time-frequency feature descriptor } \\ g & \text { wavelet basis function } \\ j & \text { imaginary unit } \\ i, k, l & \text { integer indices } \\ m, n & \text { number of time and frequency points } \\ p, q & \text { moment orders } \\ r, s, t & \text { integer indices } \\ R & \text { rank of time-frequency map } \\ \text { SRA } & \text { Systems Research Aircraft } \\ \text { SVD } & \text { singular value decomposition } \\ \text { TSVD } & \text { transformed-SVD } \\ W_{g} & \text { continuous wavelet transform } \\ & \\ \tau & \text { wavelet translation time } \\ \omega & \text { radian frequency } \\ \omega_{0} & \text { wavelet peak frequency } \\ * & \text { complex conjugate transpose } \\ <> & \text { result of moment calculation }\end{array}$

\section{Introduction}

Many aerospace disciplines face the task of analyzing nonstationary signals in which the frequency content changes with time. Most physical phenomena from acoustics, aerodynamics, thermodynamics, structural dynamics, propulsion, and controls are naturally timevarying events with frequency variations, transients, and complex harmonic interactions. Requirements for adaptive nonlinear procedures are now becoming more essential components of modern data analysis of aerospace systems in concordance with numerous other engineering and scientific applications.

A fundamental objective in data analysis of physical systems is to obtain accurate representation of the dynamics for a particular analysis. Optimal and relevant representations are desired for efficiency and consistency between the data decomposition and the physical system $[4,28,31]$. Methods for achieving this goal are often based on entropy or other statistical measures for best-basis decompositions and optimal performance ${ }^{[22,42]}$. 
Parsimonious representation of data has many such implications in denoising, compression, information retrieval, detection, identification, classification, and pattern recognition ${ }^{[8]}$. This paper emphasizes applications in dynamics analysis of aeroelastic and aeroservoelastic systems from test data. Dominant and important features in the data are extracted to provide automated information retrieval for system identification. The data analysis becomes an integral part of the system identification procedure for tracking modal stability estimates in a nonstationary setting.

Time-frequency analysis provides a powerful tool for the analysis of nonstationary signals ${ }^{[18]}$. Applications have been demonstrated in music signal analysis ${ }^{[34]}$, machinery diagnosis ${ }^{[26]}$, damage detection ${ }^{[40]}$, seismic monitoring ${ }^{[19]}$, medical signal analysis ${ }^{[41]}$, and acoustic and speech processing ${ }^{[32]}$. Transfer functions and modal parameter estimates derived from time-frequency representations have been applied to estimate state-space aeroservoelastic models $[2,3,10]$.

Time-frequency structure is revealed by quantifying the distribution of signal energy as a joint function of time and frequency. Localization of the energy density describes energy density concentrations at specific locations in the time-frequency plane. Dominant and important concentrations need to be accurately discriminated to provide descriptors relating to the location in time, time duration, frequency location, and local bandwidth of principal energy density $[11,17,43]$. Minimizing the number of these descriptors while preserving salient information from the energy distribution for modal estimation is desired for efficient dynamics analysis.

Many approaches have been proposed to extract a signal from noise in the time-frequency representation ${ }^{[33]}$. One approach performs a masking operation on the time-frequency map, then separates out desirable features $[2,10]$. Often the regions of the signal are wellconcentrated relative to the widely-distributed noise so this method works well in general. However, it requires user intervention unless regions are well-defined apriori. For this reason, an automated filtering procedure is proposed using the singular value decomposition (SVD) of the time-frequency data ${ }^{[29,43]}$.

The singular value decomposition is ubiquitous in the signal processing, identification, and control fields as a tool for rank-revealing algorithms, model reduction, subspace detection, and information retrieval $[1,20]$. The SVD is applied in this paper to derive density functions for generating moments ${ }^{[14]}$ from the joint energy density timefrequency distribution. Moments relate to the principal signatures of the nonstationary signals ${ }^{[6,27]}$. In the context of subspace estimation and information retrieval it is a data reduction for model identification.
The SVD representation tends to be sparse relative to an entire time-frequency map. The orthonormality of the SVD singular vectors for density extraction allows independent decomposition of time and frequency content in the data, thereby avoiding joint moment considerations. A new transformation of the SVD basis vectors, called the transformed-SVD (TSVD) ${ }^{[15]}$, is also used to produce more concentrated descriptions of energy density for multicomponent signals containing similar components distributed in the time-frequency plane. For separation of components requiring very fine time or frequency resolution, the TSVD may be advantageous.

Multiresolution wavelet signal processing has shown promise for studying time-frequency characteristics of signals by decomposing data into cells with properties of scale and frequency concentrated in time. These cells form a type of tiling and consist of Gaussian-windowed sinusoid basis functions (atoms), also known as Morlet wavelets, creating an atomic multiscale decomposition in a filter bank structure ${ }^{[44]}$. Competing requirements of time and frequency resolution, subject to the uncertainty principle [28], is accomplished with a combination of dyadic multiscale decomposition, compact orthogonality, and harmonic wavelet properties ${ }^{[23,24]}$.

This paper augments time-frequency multiscale wavelet processing with SVD filtering and wavelet-based modal parameter estimation. The contribution is a more general approach in which distinct analysis tools are merged into a unified procedure:

- multiresolution analysis with wavelet decomposition at multiple scales

- SVD techniques for information retrieval

- feature extraction from moments of densities

- modal parameter estimation from the complex Morlet basis functions

This automated nonlinear filtering procedure is first applied to linear and nonlinear two degree-of-freedom pitch-plunge wing section testbed models. Nonlinear dynamics are detected from the time-frequency map of the nonlinear system. Aeroelastic dynamics from the experimental flutter flight test drone, DAST (drone for aerodynamic and structural testing), is used to show how the input time-frequency signature can be used for automating the input-output data analysis. Data from a flutter encounter shows the capability for detecting an approaching instability from actual flight test data. Aeroservoelastic flight data from the F18 Systems Research Aircraft (SRA) is also investigated and comparisons made between the SVD and TSVD results. 


\section{Wavelet-SVD Method}

This section describes the method to construct timefrequency signal representations from a Morlet wavelet decomposition, then to use this energy distribution map to extract principal features from the SVD. Density functions derived from the SVD singular vectors generate spectral and temporal moments relating to features of the original nonstationary time series process. These moments are used for subsequent wavelet-based modal parameter estimation and system identification.

\section{Time-Frequency Wavelet Decomposition}

Much progress has been made in the development of time-frequency distributions ${ }^{[5]}$. For a time-frequency distribution $Q$ to be interpreted as a joint time-frequency energy density, it must be nonnegative and satisfy the correct time and frequency marginals for all time and frequency

$$
\int_{-\infty}^{\infty} Q(t, f) d t=|X(f)|^{2} \quad \int_{-\infty}^{\infty} Q(t, f) d f=|x(t)|^{2}
$$

where $X(f)=\int_{-\infty}^{\infty} x(t) \exp (-j 2 \pi f t) d t$ is the Fourier transform of the signal $x(t)$. Marginals $|x(t)|^{2}$ and $|X(f)|^{2}$ are the energy densities of time and frequency which are commonly called the instantaneous power and the energy density spectrum, respectively. Algorithms for constructing proper positive distributions satisfying these properties have recently been developed $[16,37]$. The joint energy density specifies concentrations of energy in time and frequency.

Feature extraction from joint distributions proceeds from the joint time-frequency moments of signal $x(t)$ (assumed to have unit energy) given by

$$
\begin{gathered}
\left\langle t^{p} f^{q}\right\rangle=\int_{-\infty}^{\infty} \int_{-\infty}^{\infty} t^{p} f^{q} Q(t, f) d t d f \\
p, q=1,2,3, \cdots
\end{gathered}
$$

which defines the temporal and spectral moments

$$
\begin{aligned}
& \left\langle t^{p}\right\rangle=\int_{-\infty}^{\infty} t^{p}|x(t)|^{2} d t \\
& \left\langle f^{q}\right\rangle=\int_{-\infty}^{\infty} f^{q}|X(f)|^{2} d f .
\end{aligned}
$$

In the present application, the time-frequency distribution, $Q(t, f)$, is constructed from a multiscale wavelet transform ${ }^{[3]}$. Therefore, the distribution is not the strict form of a joint energy density since the requirement on inarginals is not enforced. The wavelet transform tends to spread the energy over time and frequency, yet the wavelet decomposition is essential for identification of modal dynamics as will be shown. This energy density approximation is therefore relevant for this problem.
Multiscale transforms are used to exploit multiresolution analysis, or redundant representations of a signal on multiple frequency bands. Nonorthonormal Morlet wavelets are approximated with harmonic-like discretizations on wavelet scales corresponding to subharmonics representing overlapping frequency bands per octave (where each octave is a doubling of resolution, or a scale twice as small). These wavelets form a nonorthogonal redundant basis for the signal space ${ }^{[38]}$. Adjustment to satisfy the competing requirements of time and frequency resolution is accomplished with a combination of compact orthogonal and harmonic wavelet properties ${ }^{[23,24]}$.

A discrete wavelet transform (DWT) is derived from the wavelet basis to get a multiresolution analysis of the sampled continuous Morlet transform. Fast algorithms are realized with a dyadic multiscale decomposition. Therefore, the DWT is implemented as a dyadic filter bank covering a pre-defined range of frequencies with corresponding number of frequency bands per octave (voices/octave) ${ }^{[44]}$. Voices can be viewed as fractional dilations of a single wavelet at a particular scale. Approximate orthogonality is imposed in the multiresolution representation of the Morlet filters. Multiscale Morlet DWTs provide efficient and flexible means for analysis of nonstationary data with adjustable frequency resolution versus time localization.

The continuous wavelet transform of signal $x(t)$ over the time-scale $(\tau, a)$ plane is represented as

$$
W_{g}(\tau, a)=\frac{1}{\sqrt{a}} \int x(t) g^{*}\left(\frac{t-\tau}{a}\right) d t
$$

where scale parameter $a$ is proportional to the duration and inversely proportional to the peak frequency $\omega_{o}$ of the complex Morlet wavelet

$$
g(t)=\frac{1}{\sqrt{2 \pi}} e^{-\frac{\epsilon^{2}}{2}} e^{j \omega_{0} t}
$$

The spectrum of a dilated and translated Morlet wavelet

$$
G_{a, \tau}(\omega)=e^{-\left(\alpha \omega-\omega_{0}\right)^{2}} e^{j \omega \tau}
$$

reaches a maximum value at $a=\frac{\omega_{0}}{\omega}$. The continuous Morlet basis functions are discretized for a DWT filter bank decomposition. Signal power is preserved by enforcing the following identity with the DWT $\left(C_{w}\right.$ is an admissibility constant)

$$
\int|x(t)|^{2} d t=\frac{1}{C_{w}} \int d \tau \int_{0}^{\infty}\left|W_{g}(\tau, a)\right|^{2} \frac{d a}{a}
$$

from which the instantaneous power is expressed in terms of the wavelet time variable, $\tau$, as

$$
|x(\tau)|^{2}=\frac{1}{C_{w}} \int_{0}^{\infty}\left|W_{s}(\tau, a)\right|^{2} \frac{d a}{a} .
$$

This time-scale decomposition of data is often called a scalogram, or the energy density $\left|W_{g}(\tau, a)\right|^{2}$ of the signal over the $(\tau, a)$ plane ${ }^{[28]}$. 
As an example of a bank of discretized finite impulse response Morlet filters, figure 1 shows an array of wavelet filters for a number of voices per octave. These filters are adjusted on the plot to line up the peak magnitudes at the same locations in time. Center frequency increases with voice number, and the filters are normalized to have the same magnitude in the frequency domain at their respective peak frequencies.

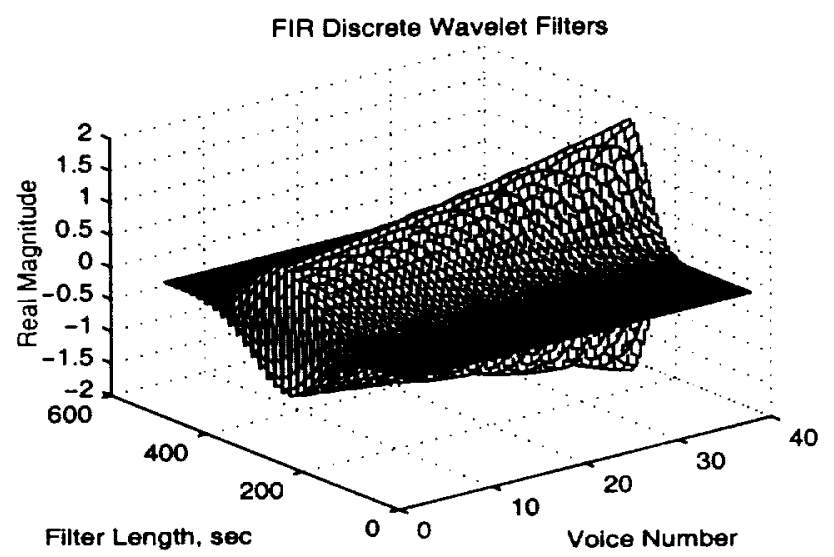

Figure 1: Wavelet filters for a number of voices per octave.

Morlet wavelets are being used to create the power distribution and to estimate the modal parameters. Therefore, an implicit filtering process is being performed independent of the explicit procedure of feature extraction and noise removal using the SVD and TSVD. The wavelet basis representation of the signal is a projection subspace from which modal parameters are derived.

\section{SVD Filtering}

Numerous wavelet thresholding techniques exist based on statistical measures of significance for denoising. These include entropy measures from information and communication theory. They are generally based on regularity, smoothness, or a low-pass character of the underlying signal relative to noise, and the compromise is between goodness-of-fit and smoothness. Because this approach is somewhat subjective by nature, a more deterministic approach is preferable. In aeroelasticity and aeroservoelasticity, the statistical criteria are generally not applicable since the dynamics can be of significant bandwidth and include high-frequency transients.

Assume the time-frequency distribution is a positive distribution in the form of a scalogram, spectrogram, or any other representation such as the Wigner-Ville distribution. In the present application the basis functions are the discretized complex Morlet wavelets, so the scalogram magnitude is appropriate. Discretized wavelets form the multiresolution analysis which can be exploited at different scales for information extraction by the SVD.
The SVD spectrum encodes the features of timebandwidth product, frequency-time dependence, and number and location of signal components. It is invariant to shifts of the signal in time or frequency $[29,43]$. While this SVD method is described for the entire timefrequency distribution, it could also be used to analyze the different scales independently or jointly.

Consider the distribution $Q$ as an $m$-by- $n$ discrete matrix $A$ such that $A(k, l)=Q\left(t_{k}, f_{l}\right)$, where $k=1,2, \cdots, m$ and $l=1,2, \cdots, n$ for $m$ time points and $n$ discrete frequencies. This matrix can always be decomposed with the SVD into a set of basis matrices $A_{i}$ with corresponding singular values, or weights, $\sigma_{i}$

$$
A=\sum_{i=1}^{R} \sigma_{i} A_{i} ; \quad A_{i}=u_{i} v_{i}^{*} .
$$

$R$ is the rank, the set of constant weights $\sigma_{i}$ are ordered such that $\sigma_{1} \geq \sigma_{2} \geq \cdots \geq \sigma_{R}>0$, and each $A_{i}$ (or $\sigma_{i}$ ) corresponds to singular vectors $u_{i}$ and $v_{i}$ as shown. Time and frequency aspects of the $A_{i}$ are independent since $u_{i}$ and $v_{i}$ are orthonormal. The maximum value of the rank $R$ can only be as large as the minimum of the rowcolumn $(m-n)$ dimension of $A$. The objective is to get a reduced set of energy concentrations defined by the $A_{i}$ to generate time-frequency features based on the relative magnitudes of the associated $\sigma_{i}$. Note that the rank $R$ can never exceed the number of discrete frequencies $n$, i.e., $R \leq n$, since there will always be more time points than discrete frequencies.

The matrix $A$ is used with the weights to extract highlights from the wavelet map by characterizing the $A_{i}$ with the joint distribution of equation 1 . For each $\sigma_{i}$, a corresponding region in the distribution is desired $\left(A_{i}\right)$ which is weighted in value by the magnitude of $\sigma_{i}$ relating to its respective information content. From the original joint moments of equation 1, the SVD separates the distribution into $R$ primary moments. This decomposition correlates directly with independent time and spectral moments expressed in equation 2 through independent singular vectors $u_{i}$ and $v_{i}$ in equation 3 , so joint moments need not be considered ${ }^{[14]}$.

A unit-energy positive distribution $A$ matrix does not guarantee that each $A_{i}$ will be element-by-element positive, which is necessary for a proper density function. Matrices $\tilde{A}_{i}$ are constructed from the $A_{i}$ by squaring each matrix element to create proper densities for moment calculations. Each $\tilde{A}_{i}$ is formed from the elementby-element square of the associated singular vectors $u_{i}$ and $v_{i}$, and the corresponding squared vectors are denoted by $\tilde{u}_{i}$ and $\tilde{v}_{i}$. The new basis matrices are

$$
\tilde{A}_{i}=\tilde{u}_{i} \bar{v}_{i}^{*} \geq 0 \text {. }
$$

Density functions $\tilde{u}_{i}$ and $\tilde{v}_{i}$ compose proper density functions $\bar{A}_{i}$ by being positive and orthonormal, and by be- 
ing independent, they are also the time and frequency marginals of the respective $\tilde{A}_{i}$. Moments of the density functions constructed from the singular time and frequency vectors are used to formulate features. Temporal and spectral moments corresponding to equation 2 are calculated as

$$
<t^{p}>_{i}=\sum_{k=1}^{m}\left(t_{k}\right)^{p} \tilde{u}_{i}(k) ; \quad<f^{q}>_{i}=\sum_{l=1}^{n}\left(f_{l}\right)^{q} \tilde{v}_{i}(l) .
$$

First moments $(p=q=1)$ estimate time-correlated instantaneous frequency, and the second moments ( $p=$ $q=2$ ) determine the time duration and instantaneous bandwidth for each feature in the distribution. A feature descriptor $F_{i}$ is therefore defined by

$$
\begin{gathered}
F_{i}=\left(\hat{\sigma}_{i}, \bar{t}_{i}, \bar{f}_{i}, \hat{t}_{i}, \hat{f}_{i}\right) \\
\equiv\left(\frac{\sigma_{i}}{\sigma_{1}},\left\langle t>_{i},\left\langle f>_{i}, \sqrt{\left\langle t^{2}>_{i}-\bar{t}_{i}^{2}\right.}, \sqrt{\left\langle f^{2}>_{i}-\bar{f}_{i}^{2}\right.}\right) .\right.\right.
\end{gathered}
$$

Each feature descriptor $F_{i}$ for each $A_{i}$ is a 5-element vector which includes the normalized singular value weight $\hat{\sigma}_{i}$, time location $\bar{t}_{i}$, instantaneous frequency $\bar{f}_{i}$, time duration $\hat{t}_{i}$, and instantaneous bandwidth $\hat{f}_{i}$, of the feature. Features are $\hat{\sigma}_{i}$-scaled rectangle regions in the timefrequency map. They are used as windows on the original scalogram to extract regions for further analysis.

The number of desired features should preferably be as small as possible while preserving relevant dynamics. The separation property of the SVD helps maintain important information with a minimal number of features. Generalizing the same process to higher-order moments gives instantaneous skew and kurtosis ${ }^{[6]}$ which may reveal data anomalies, asymmetries, and nonlinearity.

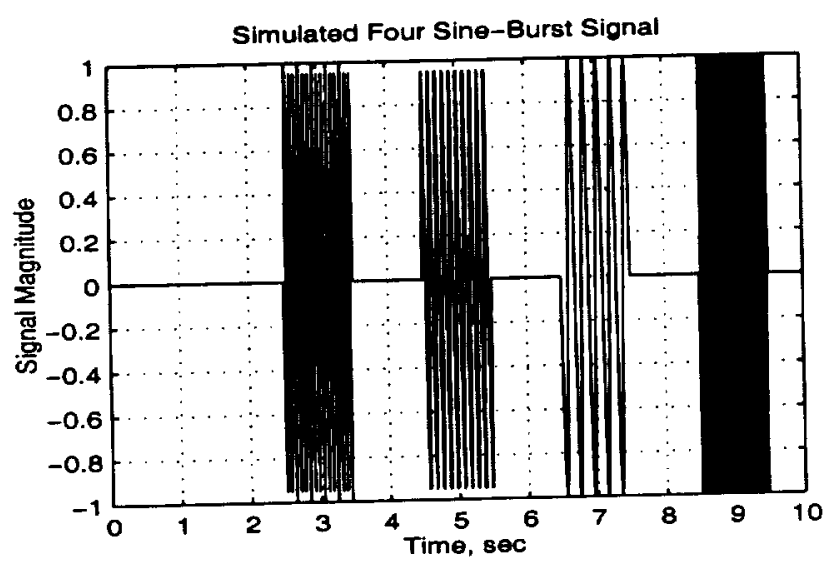

Figure 2: Four sine-bursts of equal amplitude.

A simple example of a simulated analysis with SVD filtering uses the signal in figure 2 . There are four sine-bursts at $15,10,5$, and $25 \mathrm{~Hz}$, respectively. A wavelet decomposition and SVD filtering procedure is performed on this signal to produce the plots in figure 3 . The top plot represents an energy density distribution contour (plotted in two dimensions) of the original wavelet coefficients. The middle plot represents the sum of all rectangular features $F_{i}$ determined from the SVD filter. The bottom plot accentuates the middle plot using a log-scale (dB) for the out-of-plane contour dimension. A log-scale region will cover at least as much as the linear scale contour of the middle plot, and all contours are normalized to their maximum magnitude. The middle and bottom plots are identical except for the contour scale.
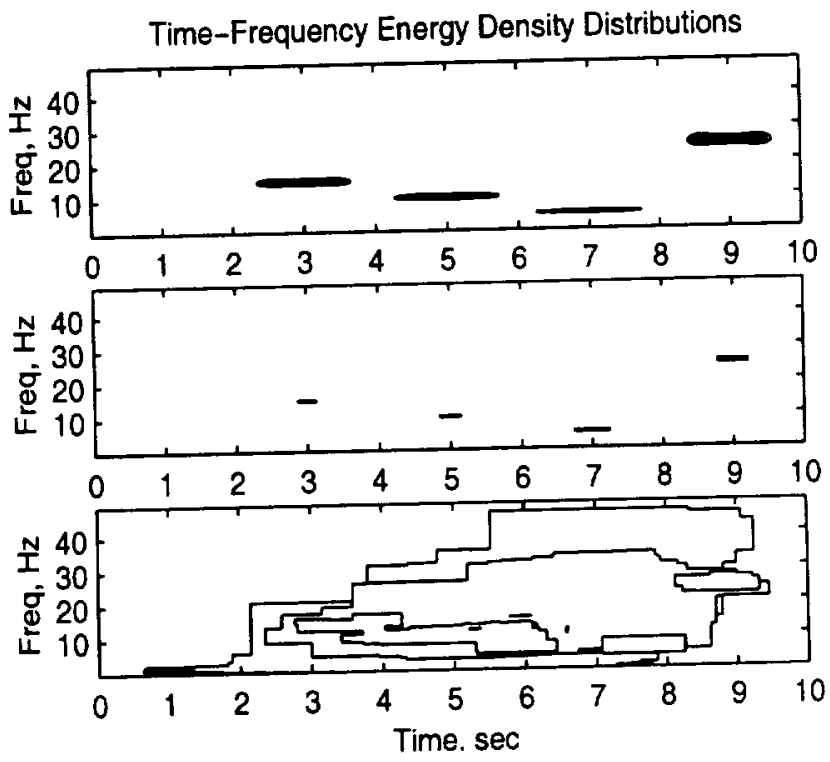

Figure 3: Contour plots from original wavelet decomposition (top), SVD-filtered (middle), and SVD-filtered (bottom, log-scale) scalograms of four sine-burst signal.

Note the more compact representation from SVD filtering (middle plot) compared to the original decomposition. For an automated analysis, we choose the order of the distribution to be the full rank $R$. Of the total 300,000 elements in the distribution, the rank in this case reduces the number of features to $R=300$, resulting in a dramatic data reduction for model identification.

\section{Transformed-SVD}

A potential problem with the standard SVD filter stems from the information contained in the individual $A_{i}$ terms of the decomposition. The principal information is ordered according to values of $\sigma_{i}$, but often there are multiple time-frequency regions with similar energy density magnitudes which will contribute equally to each $A_{i}$. Moments calculated from the singular time and frequency vectors contain simultaneous contributions from distributed regions since the vectors span the entire scalogram. Information is not concentrated because the SVD cannot separate energy density concentration in the individual $A_{i}$ so the features consist of linear combinations of many singular vectors. 
Feature extraction would benefit from more localized signal content in each $A_{i}$ for two reasons. First, for a fixed number of features, fewer terms in the SVD would enhance specific localized content. Second, for a fixed number of terms, higher fidelity features would be produced. Therefore, what is desired is a rotation of the singular basis to minimize the number of basis vectors to interpret each density concentration. Ideally, each $A_{i}$ term should relate to a single localized concentration.

This problem can be posed as follows: find an orthonormal transformation for the $u_{i}$ and $v_{i}$ vectors such that the means are maximized in the new basis. The attempt is to concentrate the densities into smallest regions in the plane. For unknown transformation $C$, and orthonormality condition $C C^{*}=C^{*} C=I$, the means are expressed in terms of the $c_{i k}$ coefficients. Orthonormality implies that $\sum_{r=1}^{m} y_{i}^{2}(r)=1$, so the means of the transformed vectors $y_{i}=\sum_{k} c_{i, k} u_{k}$ are the first moments $\left\langle y_{r}\right\rangle$ given by

$$
\begin{array}{rlc}
\left\langle y_{r}>_{i}\right. & = & \sum_{r=1}^{m} r y_{i}^{2}(r) \\
& = & \sum_{r=1}^{m} r \sum_{s=1}^{R} c_{s, i} u_{s}(r) \sum_{t=1}^{R} c_{t, i} u_{t}(r) \\
& = & \mathbf{c}_{i}^{*} \mathbf{M c}_{i}
\end{array}
$$

In this quadratic form, the unique solution for maximizing the means of the $y_{i}$ vectors is achieved when the $c_{i}$ are the eigenvectors of the $\mathbf{M}$ matrix. Similarly, an orthonormal matrix $\mathrm{D}$ is found independently for the vectors $v_{i}$ by maximizing the means of vectors $x_{i}=\sum_{k} d_{i, k} v_{k}$. Compared to the standard SVD, the transformed-SVD is as follows [15]

$$
A=U S V^{*} ; \quad U=Y C^{*} ; \quad V=X D^{*}
$$

and the proper substitutions made to get the TSVD

$$
A=\left(Y C^{*}\right) S\left(X D^{*}\right)^{*}=Y\left(C^{*} S D\right) X^{*}=Y Z X^{*} .
$$

Now that instead of just a sequence of singular values $\sigma_{i}$ from the $S$ matrix of the SVD, there exists a full singular matrix $Z=C^{*} S D$ of terms to weight the new singular vectors $X$ and $Y$ in the TSVD. These terms have the same function as the terms in $S$, but instead of correspondence to linear combinations of concentrations in $u_{i}$ and $v_{i}$, the Z-elements, $z_{i k}$, correlate to transformed singular vectors $y_{i}$ and $x_{k}$. Obviously there are significantly more $\left(m\right.$-by-n) of the $z_{i k}$ than the $\sigma_{i}(R \leq n)$. The singular weights in $Z$ are ordered and the new TSVD decomposition for the first $R$ of the $i k$-components takes the form (where now $B \neq A$, compare to equation 3 )

$$
B=\sum_{i=1}^{R} z_{i k} B_{i} ; \quad B_{i}=y_{i} x_{k}^{*}
$$

Returning to the signal from figure 2, a transformedSVD analysis is performed and plotted in figure 4 for
Time-Frequency Energy Density Distributions
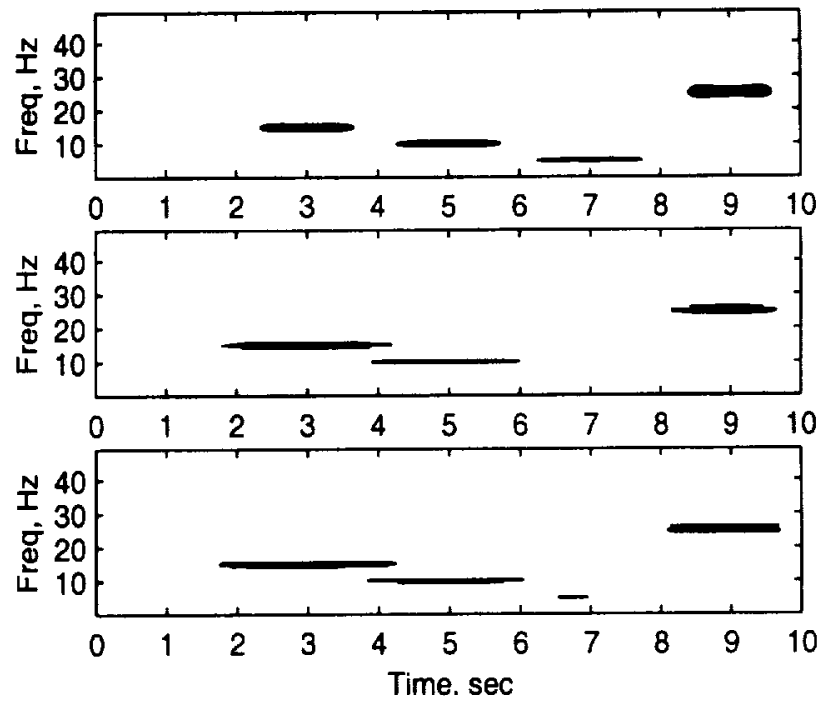

Figure 4: Contour plots from original wavelet decomposition (top), TSVD-filtered (middle), and TSVD-filtered (bottom, log-scale) scalograms of four sine-burst signal.

comparison with the SVD result in figure 3 . The plots in figure 4 correspond to those in figure 3 , so the top plot is identical. The middle TSVD contour plot in figure 4 shows an enhanced version of the plot in figure 3 by extension of the time-range estimate of three of the sine-bursts. Note the longer feature window based on the computed $F_{i}$ compared to the original distribution contour. The burst at $5 \mathrm{~Hz}(7 \mathrm{sec})$ is not distinguishable in the TSVD middle plot, but in the remarkably similar bottom log-scale plot it reappears. This burst at $5 \mathrm{~Hz}$ has the least total energy content.

Observe that in the bottom two plots, the TSVD has remarkably more detail with the same number $(R=300)$ of singular vectors. Information extraction is devoted to the regions of the sine-bursts, and not spread over unnecessary areas of the time-frequency plane as in the case of the SVD in the bottom plot of figure 3. The more compact representation from the TSVD will improve further analysis by concentrating on the important regions of interest for modal identification.

It was already shown in a previous study ${ }^{[15]}$ how the TSVD separates and improves the quality of the timefrequency features with a small set of descriptors for classification purposes. The present application for flight data analysis emphasizes the filtering aspects of the TSVD versus the SVD. The purpose is not to find a minimum number of descriptors, but to get the highest quality filtered output for a fixed number of features determined by the rank, $R$, of the discretized time-frequency map. This will allow an automated procedure for preprocessing with a time-frequency filter to augment modal parameter estimation and system identification. 
SVD approaches are attractive from a systems analysis perspective. Prevalence in model reduction, subspace identification, and optimal filtering point to its significance as a tool for information retrieval. Some of its power lies in the geometry of the singular vectors as a distance measure between subspaces $[7]$. Also, for on-line implementations, popular SVD recursive procedures allow for adaptive signal processing by using fast updatingdowndating schemes ${ }^{[1,9]}$. The idea is to perform the wavelet decompositions, update the time-varying distributions, and apply on-line procedures for the analysis.

\section{Parametric Modal Estimation}

Modal parameters can be estimated with wavelets by analysis of the system impulse response ${ }^{[3,36]}$ (see appendix). The DWT of a signal using the complex Morlet wavelet is a complex-valued matrix whose modulus and phase are related to impulse response parameters. Linear phase variation of the DWT estimates the instantaneous frequency. Wavelet modulus decay is used similarly to derive decay rate for corresponding modal damping estimates assuming the instantaneous frequencies correspond to modal dynamics. In the current application, this procedure is applied at every time point assuming at each instant that the response is a sum of multiple degree-of-freedom impulse responses.
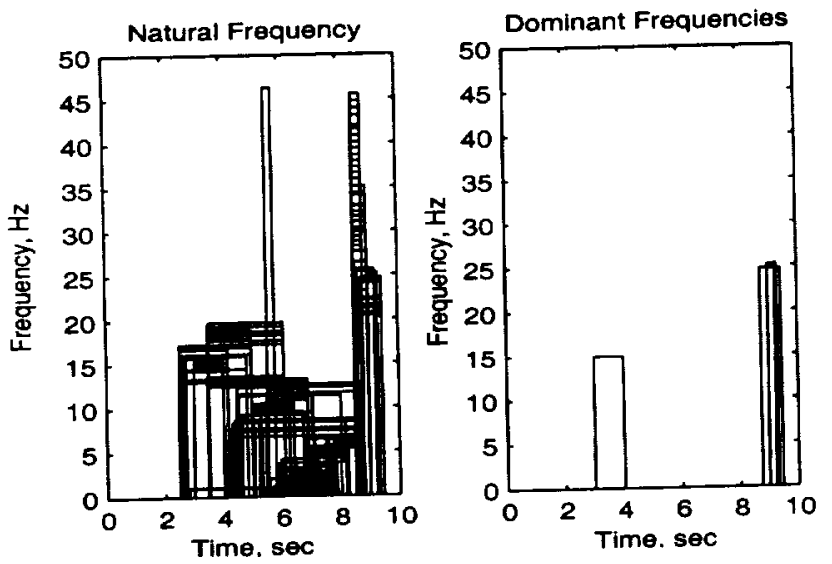

Figure 5: Instantaneous frequency estimates (left) and detected dominant spectrum (right) using the SVD.

Instantaneous frequency estimation of the four sine-burst signal of figure 2 is illustrated in figures 5 and 6 using the SVD and TSVD, respectively. The plots show frequency estimates over the corresponding detected period of time from the DWT. Right plots titled Dominant Frequencies in each figure are subsets of the those titled Natural Frequency. Left plots designate raw estimates from the respective filtered regions, and right plots refer to the spectrum detected by using a threshold parameter on the wavelet coefficients. This threshold is 0.5 of the normalized absolute magnitude of the scalogram. Threshold estimates are referred to in this report as dominant.
In the SVD analysis of figure 5, numerous frequencies are detected from the extracted regions of figure 3 , but of those only two dominant frequencies at $15 \mathrm{~Hz}(3 \mathrm{sec})$ and $25 \mathrm{~Hz}(9 \mathrm{sec})$ in the right plot are estimated based on this threshold. The congested left plot indicates that many detected frequencies are redundant or spurious estimates from large relatively insignificant regions of the timefrequency map (bottom plot of figure 3). Conversely, in figure 6 for the TSVD, the left and right plots are identical and all the frequencies are detected as being dominant. This demonstrates that the TSVD is detecting only that part of the spectrum that is significant in the data without any threshold criteria.
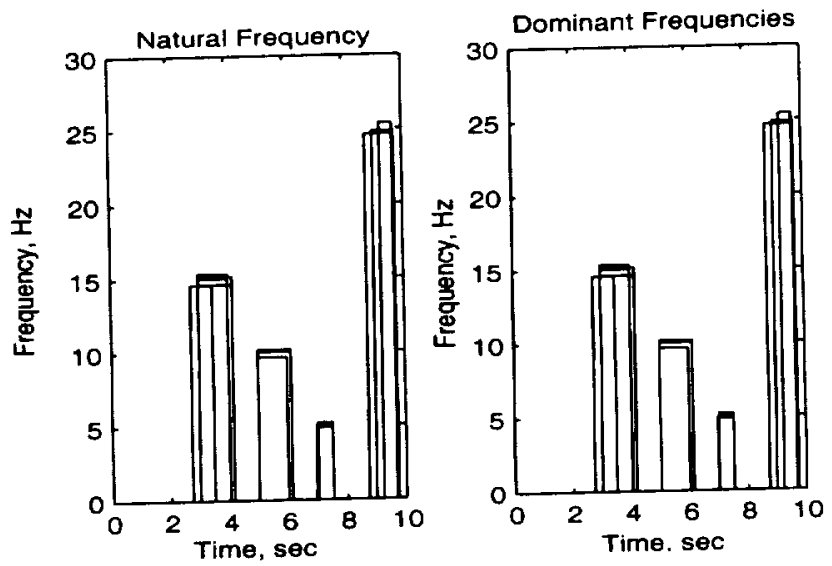

Figure 6: Instantaneous frequency estimates (left) and detected dominant spectrum (right) using the TSVD.

\section{Aeroelastic Testbed Model}

Previous studies have investigated applying wavelets for analysis of structural and aeroelastic nonlinear systems $[25,30,45]$. SVDs have been used to extract the minimum embedding dimension from noisy nonlinear systems ${ }^{[39]}$. In this section the wavelet-SVD approaches are applied to investigate linear and nonlinear reponses for a simple aeroelastic system. An aeroelastic testbed has been developed at Texas A\&M University for flutter research using a prototypical aeroelastic wing section [12]. This quasisteady pitch-plunge system is described by two-degree-of-freedom aeroelastic equations of motion. A control surface configuration was used for closed-loop control ${ }^{[21]}$.

Nonlinearity is introduced to the system dynamics through the stiffness associated with pitch. This stiffness is described by a nonlinear polynomial function of the pitch angle. Such structural nonlinearities occur in physical aeroelastic systems and have been investigated to determine their effect on inducing limit cycle oscillations. The two parameters that determine the response of the wing and the onset of limit cycle oscillations. are the elastic axis location and the freestream velocity. The 

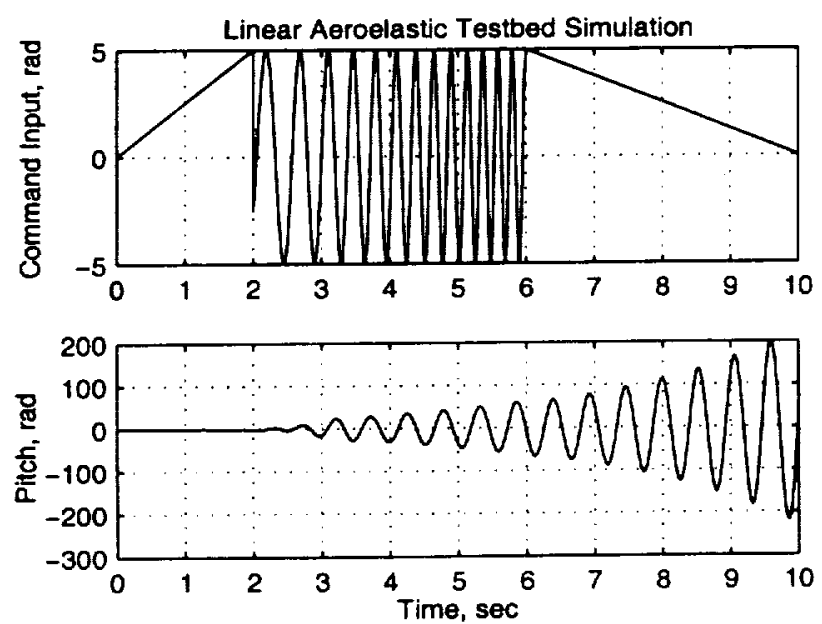

Figure 7: Chirp control command input (top) and pitch response (bottom) from linear aeroelastic testbed model.

stiffness functions associated with the pitch degree of freedom are chosen here to represent a linear spring and a nonlinear hardening spring.
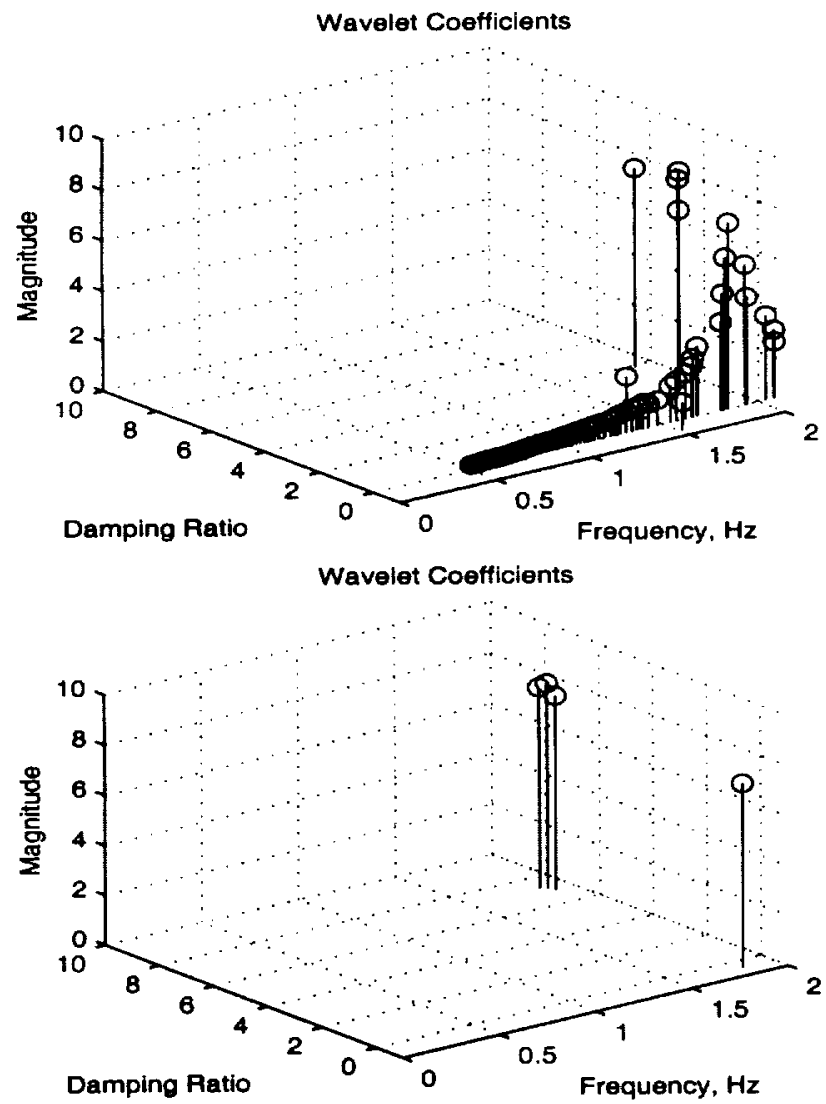

Figure 8: Modal parameter estimates from testbed linear simulation using SVD (top) and TSVD (bottom).

A classical flutter instability is encountered for the linearized dynamics near a speed of $12 \mathrm{~m} / \mathrm{s}$ at about $2 \mathrm{~Hz}{ }^{[25]}$. Figure 7 represents a control input chirp of
$0-5 \mathrm{~Hz}$ (top) and the unstable pitch response at $12 \mathrm{~m} / \mathrm{s}$ (bottom). Both SVD and TSVD results are presented in figure 8 . These plots represent the estimates and corresponding wavelet coefficient magnitudes from which the estimates were derived. Magnitudes of the coefficients determine a relative level of significance, or measure of observability. The upper plot from the SVD shows the instability detected near $2 \mathrm{~Hz}$. The array of zero damping values at frequencies below the unstable mode indicate an output tracking of the input (disguised by the magnitude in figure 7) until an instability is detected at $1.5 \mathrm{~Hz}$ near the pitch mode natural frequency. TSVD estimates of the same instability are more accurate near $2 \mathrm{~Hz}{ }^{[25]}$ with much fewer spurious estimates.

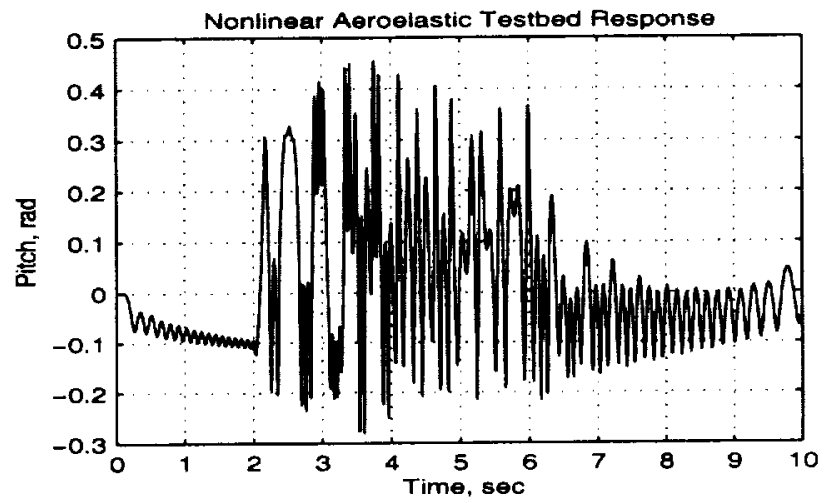

Figure 9: Pitch response from nonlinear aeroelastic testbed model chirp command input.
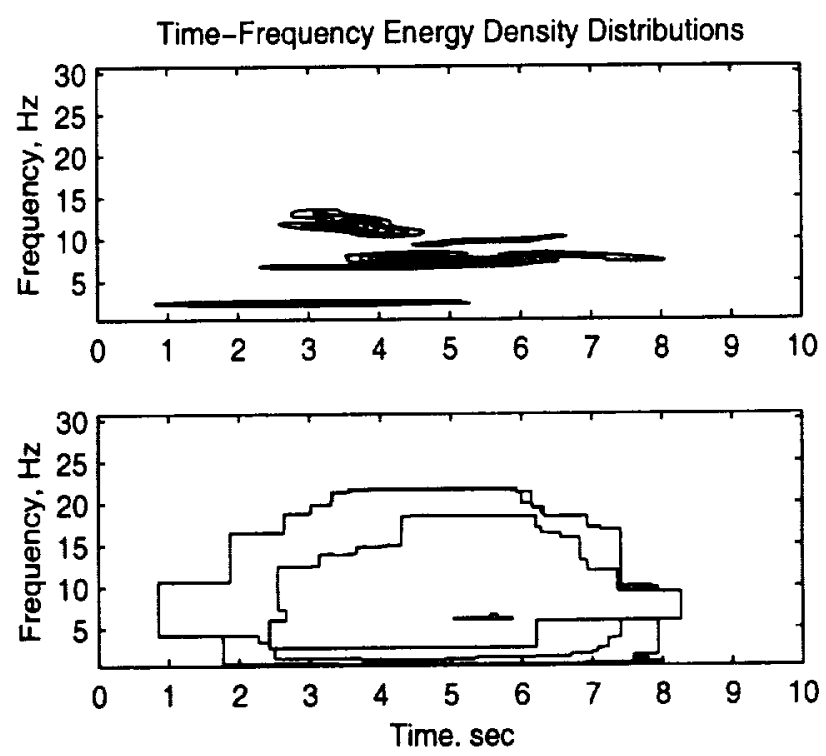

Figure 10: Contour plots from original wavelet decomposition (top) and SVD-filtered (bottom, log-scale) scalograms of nonlinear aeroelastic testbed simulation.

A nonlinear response from the same chirp input also at $12 \mathrm{~m} / \mathrm{s}$ with a nonlinear hardening spring in the pitch degree of freedom is shown in figure 9 . The corresponding wavelet scalogram and SVD-filtered contour plot are 
shown in figure 10. Note the scattering of the output spectrum from a well-defined chirp input, thus indicating the nonlinearity ${ }^{[25]}$. There is clear indication of higher harmonics from the primary $2.5 \mathrm{~Hz}$ mode at higher frequencies near $7.5 \mathrm{~Hz}$ and $12.5 \mathrm{~Hz}$ (the third and fifth harmonics). This result is also evident from the corresponding frequency and damping estimates in figure 11. From the multitude of SVD raw estimates, this plot shows only the dominant coefficients using the threshold discussed previously. At any time during the response, at least two of three of the resonances are evident.

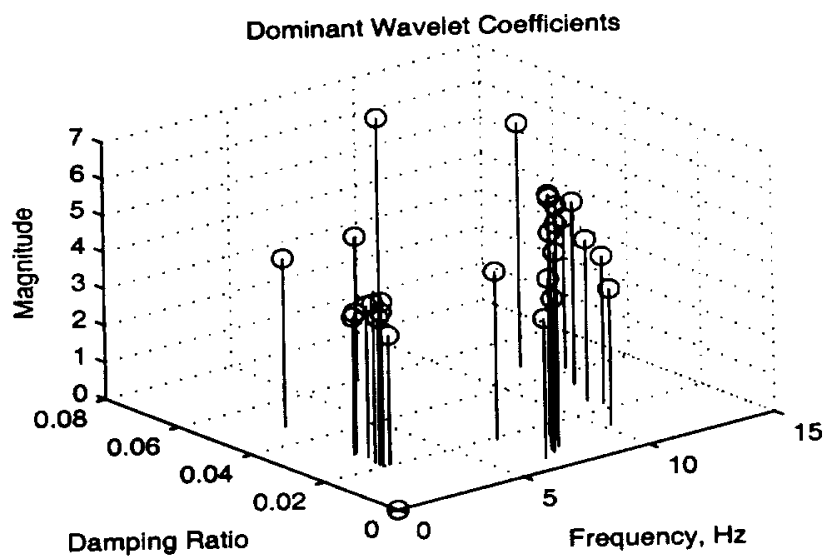

Figure 11: Modal parameter estimates from nonlinear aeroelastic testbed simulation using SVD filtering.

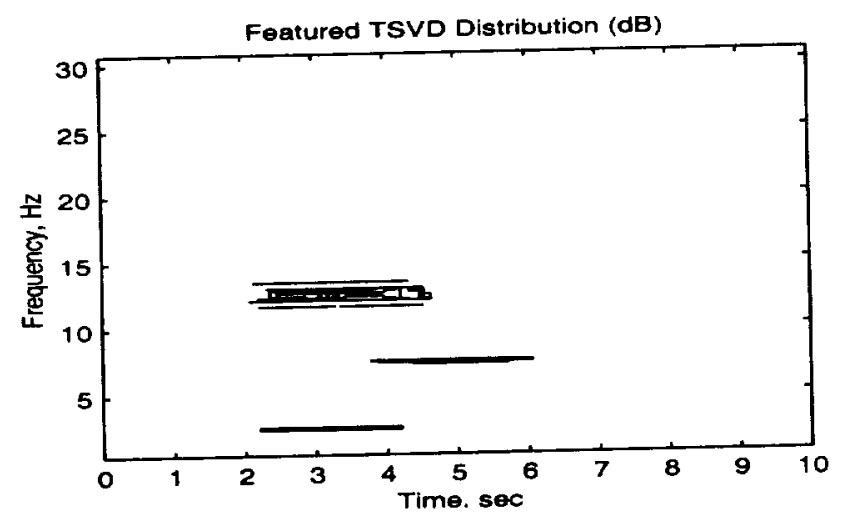

Figure 12: Contour plot of TSVD-filtered scalograms (log-scale) of nonlinear testbed simulation response.

More precise tracking of the modes from the TSVD is evident from the filtered contour plot of figure 12. This plot corresponds to the previous contours from figure 10 . High definition frequency estimation compared to the SVD is clearly seen, and results in the raw estimates plotted in figure 13 being identical to the TSVD dominant estimates (not shown).

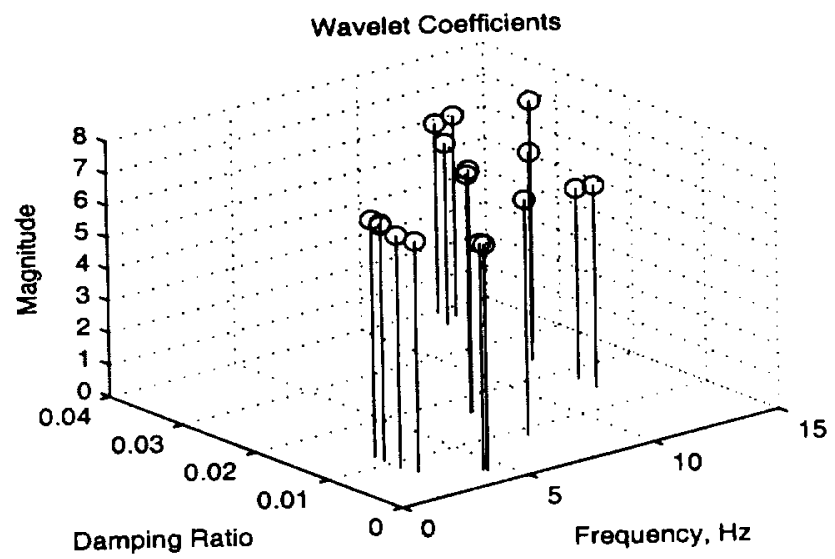

Figure 13: Modal parameter estimates from nonlinear aeroelastic testbed simulation using TSVD filtering.

\section{DAST Flutter Data Analysis}

For an automated data analysis procedure to be effective with a known input signal, it is advantageous to use the time-varying input spectrum for tracking output responses. Linear dynamics may then be discerned from unmodeled dynamics and nonlinearity outside of the input frequency range. For this purpose, using actual aircraft data, wingtip accelerometer data is analyzed from the NASA DAST ${ }^{[13]}$ (drone for aerodynamic and structural testing, figure 14). Part of the DAST program in 1980 was to pursue investigations on a drone equipped with a flutter suppression system to enable flight beyond the open-loop stability boundary. Wingtip accelerometer response data was acquired with $10-40 \mathrm{~Hz}$ logarithmic chirps and $20 \mathrm{~Hz}$ sinusoidal doublets into the aileron control surface.

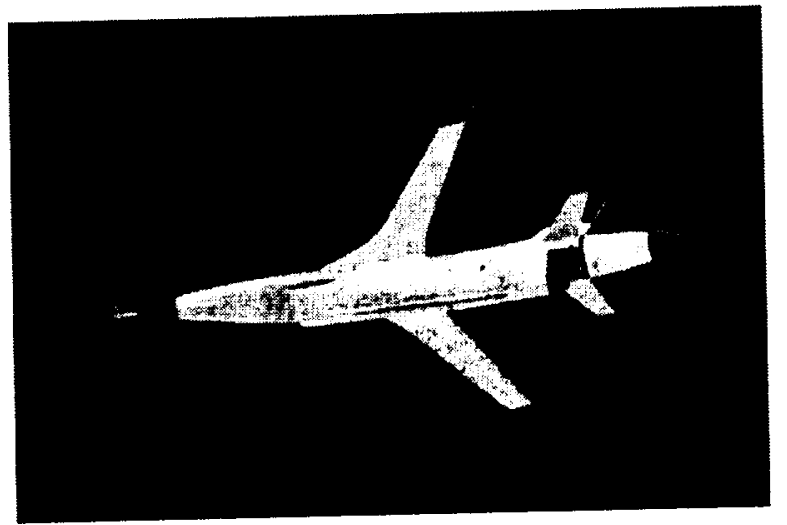

Figure 14: NASA DAST vehicle in flight.

From the time-frequency path of the input signal, a mask is created by SVD or TSVD filtering to be applied to the accelerometer response. An aileron chirp input (top) and wingtip accelerometer response (bottom) are shown in figure 15 at Mach 0.8 about a minute before the drone encountered an aeroservoelastic instability ${ }^{[13]}$. 

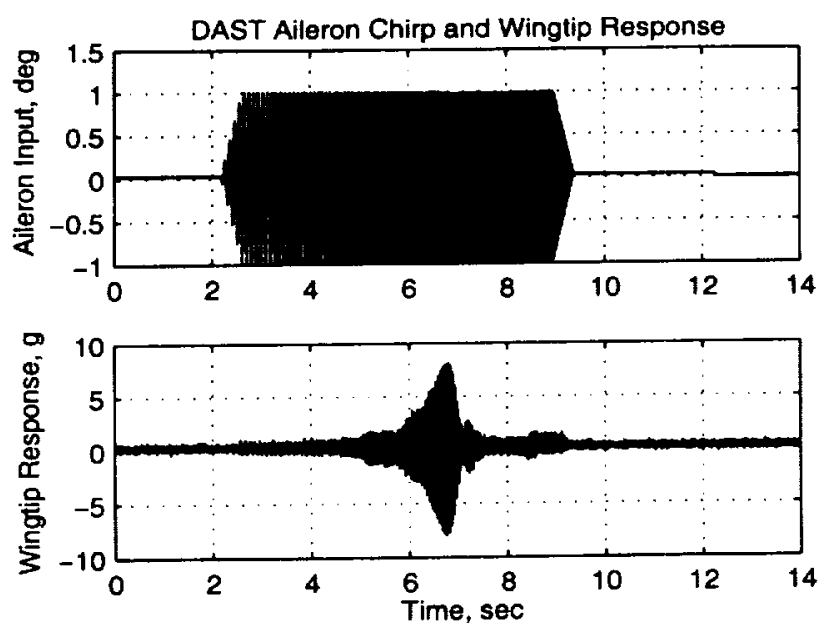

Figure 15: Aileron chirp (top) and wingtip accelerometer response (bottom) from DAST at Mach $0.8,15,000$ feet.

Figure 16 shows contour plots of the aileron chirp input. The SVD-filtered envelope (bottom plot) bounds the input more than adequately. Also, shown in figure 17 , the estimated modal frequency and damping show the primary wing bending mode near $20 \mathrm{~Hz}$, but also other minor harmonics of much smaller magnitude near $40 \mathrm{~Hz}$ and $60 \mathrm{~Hz}$. Note that in the SVD-filtered contour plot of figure 16 , the spread up to $60 \mathrm{~Hz}$ occurs when the primary $20 \mathrm{~Hz}$ mode is excited by the chirp input $(6-7 \mathrm{sec})$.
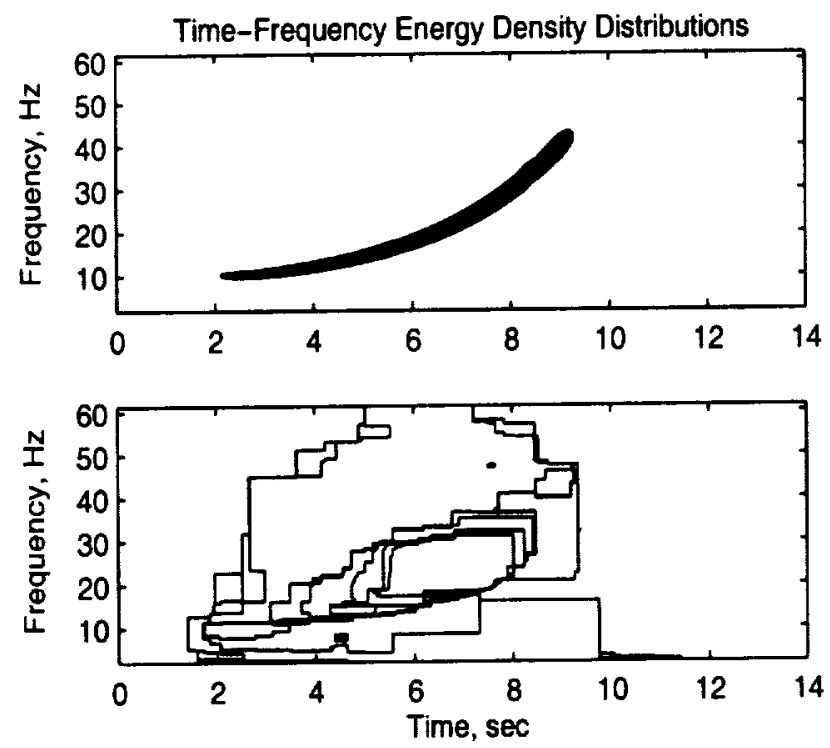

Figure 16: Contour plots from original wavelet decomposition (top) and SVD-filtered (bottom, log-scale) scalograms of DAST aileron chirp input.

In contrast, the TSVD-filtered contour of figure 18 discriminates discrete frequency bins directly along the time-varying chirp input. Dynamics outside of the timefrequency path of the input will not be observed. Estimates will only be derived locally along the input time-

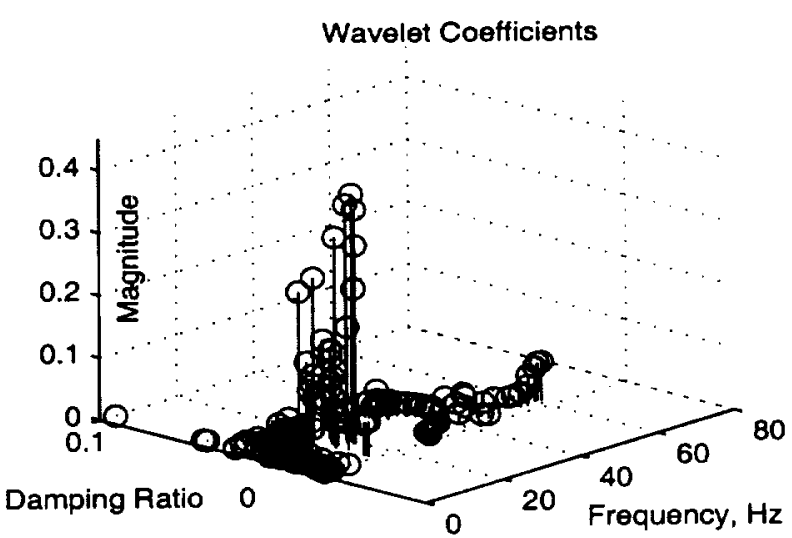

Figure 17: Modal parameter estimates from DAST wingtip acclerometer from chirp using SVD filtering.

frequency window. This property of the TSVD may be used to separate linear output response from unmodeled dynamics and nonlinearity.

The SVD filter generally encompasses a much larger region than the input alone, with the higher contours more adjacent to the dominant energy spectrum. While not as precise a filter as the TSVD, it detects a timefrequency envelope that thoroughly bounds the input. A TSVD filter concentrates more singular vector contributions (moments) into smaller areas than the SVD filter. The TSVD may actually miss some input dynamics from its moments for a reasonable order approximation comparable to the SVD. A higher order approximation is possible with the TSVD as mentioned in the comments above equation 4 , but this paper only addresses equalorder approximations between the SVD and TSVD.

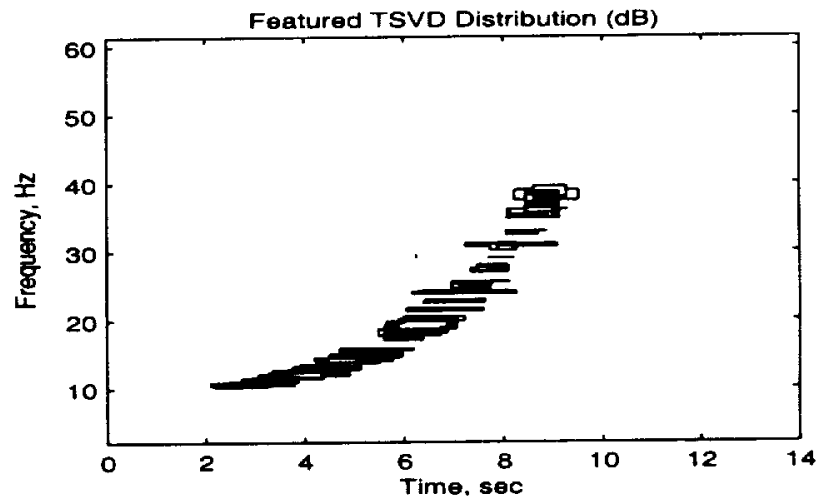

Figure 18: Contour plot of TSVD-filtered scalogram (log-scale) of DAST aileron chirp input.

Wavelet reconstructions are created from the coefficients of the time-frequency map that are retained by filtering, masking, or thresholding. In a direct filtering or masking application, the quality of the wavelet reconstruction of a time response depends on the time-frequency window used on the response. Good results are obtained when 


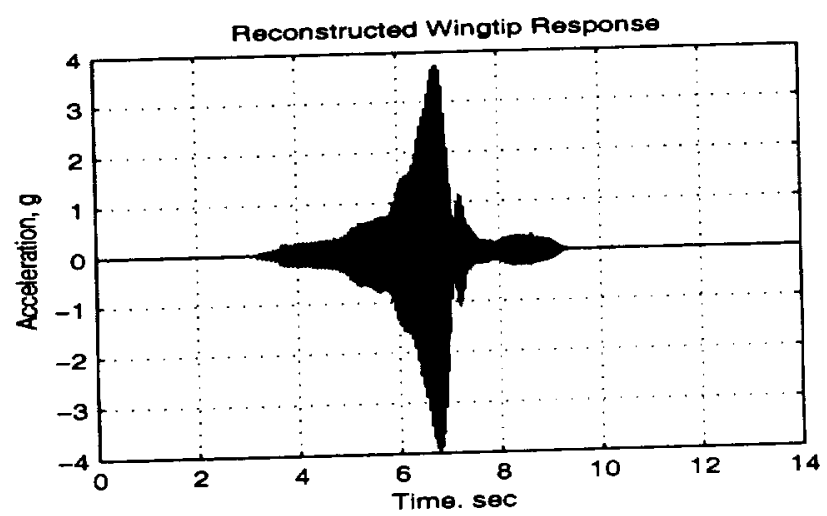

the TSVD. Both reconstructions are modulated modal responses, but the TSVD consists of narrower bandpass filters in its modulation.
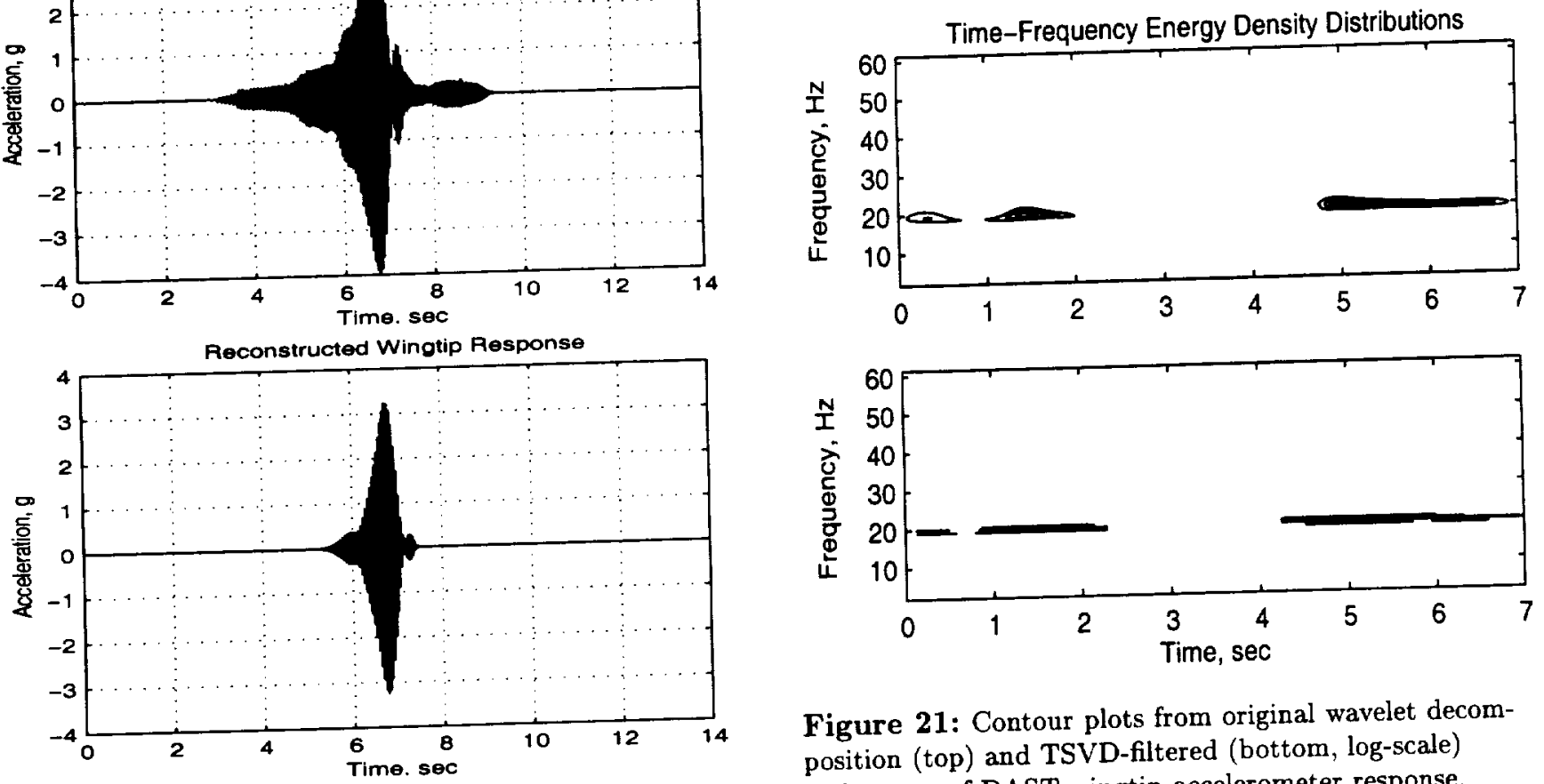

Figure 21: Contour plots from original wavelet decomposition (top) and TSVD-filtered (bottom, log-scale) scalograms of DAST wingtip accelerometer response.

Figure 19: Reconstructed SVD-filtered (top) and TSVD

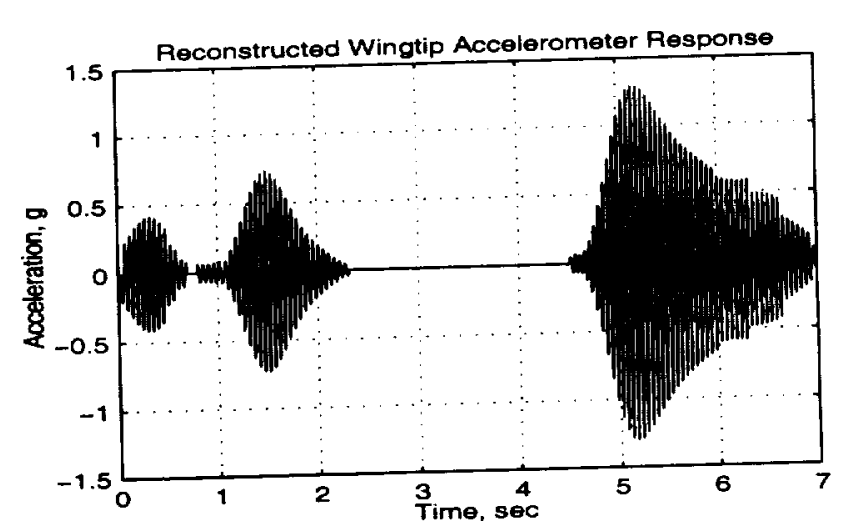

all the significant dynamics are retained. For instance, with no filtering or masking, the reconstructed response is simply a projection onto the wavelet basis functions. This signal generally resembles the original signal because of the good reconstruction properties from the redundancy in the multiscale wavelet transform.

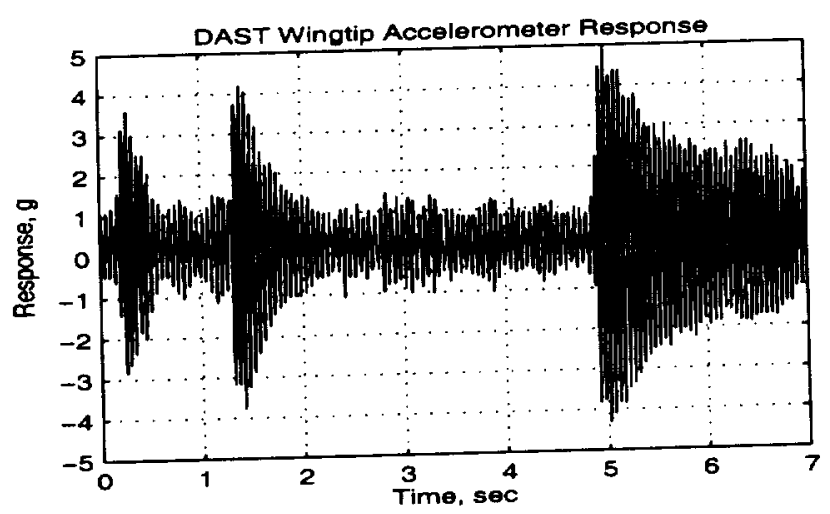

Figure 20: Wingtip accelerometer response from DAST aileron-pulsed input at Mach $0.825,15,000$ feet.

For the wingtip accelerometer response of figure 15, comparisons are made between the SVD (top) and TSVDfiltered (bottom) reconstructions in figure 19. The SVD filter covers more of the time-frequency plane, and this shows up in the reconstructed response as compared to

Figure 22: Reconstructed TSVD-filtered DAST wingtip accelerometer response at Mach $0.825,15,000$ feet.

To demonstrate the estimation procedure for an actual aircraft instability, figure 20 represents the wingtip accelerometer response at Mach 0.825 immediately before the closed-loop instability. During the last three sine pulses, the TSVD filter results are shown in the the contour plots of figure 21. The SVD filter has difficulty making a precise determination of the pulse energy density map (not shown). Tracking the pulse responses is complete and accurate with the TSVD filter.

Figure 22 represents a reconstructed TSVD-filtered version of the signal in figure 20. From the coefficients of the filter shown in figure 21, the reconstructed time response resembles an impulse response of the mode near $20 \mathrm{~Hz}$. Estimated modal frequency and damping from the 
TSVD in figure 23 shows clearly the $20 \mathrm{~Hz}$ wing bending mode and reduction in modal damping with time.
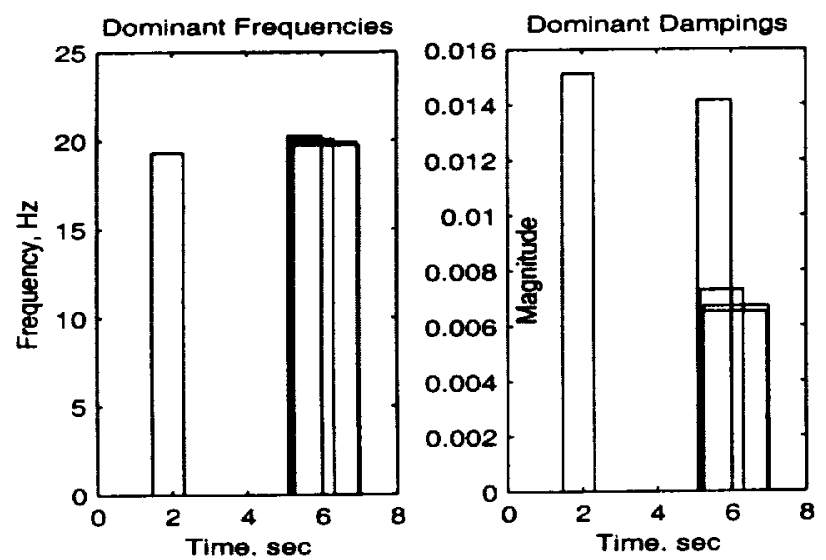

Figure 23: TSVD estimated modal frequencies (left) and damping ratios (right) from DAST wingtip accelerometer.

\section{F18 Aeroservoelastic Data Analysis}

Digital excitation signals were implemented on an the NASA F18 Systems Research Aircraft (SRA) for a baseline model update and validation effort to support the F18 Active Aeroelastic Wing ${ }^{[35]}$ program. Multisine signals were added to aileron, stabilator, and rudder actuator commands. In this example, the differential aileron command is used for excitation at Mach 0.9, 10,000 feet. Wingtip and lateral fuselage accelerometer responses are used to demonstrate the SVD and TSVD filtering process with a discontinuous input in the time-frequency plane. Multisine waveforms have a chirp-like quality, but with a burst of high-frequency chirp at the end of the signal [2].

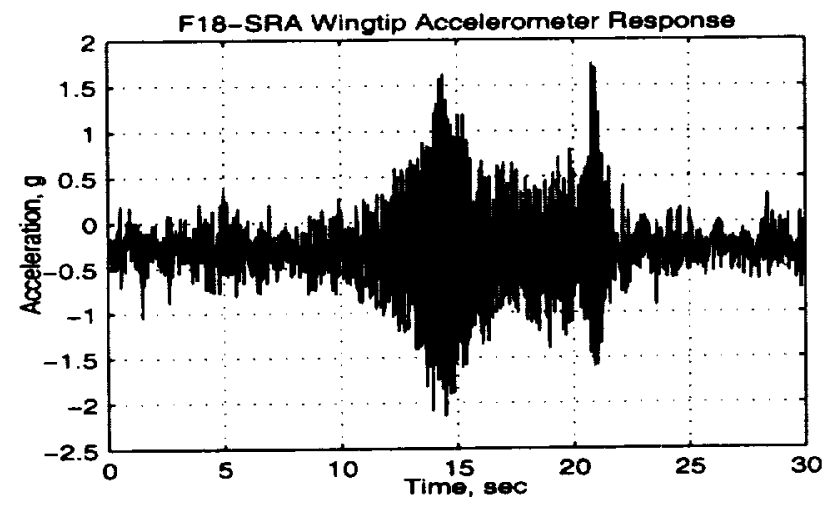

Figure 24: F18-SRA wingtip accelerometer response from a multisine aileron input.

A wingtip accelerometer response to a multisine aileron input is shown in figure 24. Figure 25 (top plot) shows the nature of a multisine in the wingtip response for a 35 to $-5 \mathrm{~Hz}$ sweep. Note the $13-15 \mathrm{~Hz}$ pulse at the end of the main sweep (21 sec), characteristic of the multisine. The
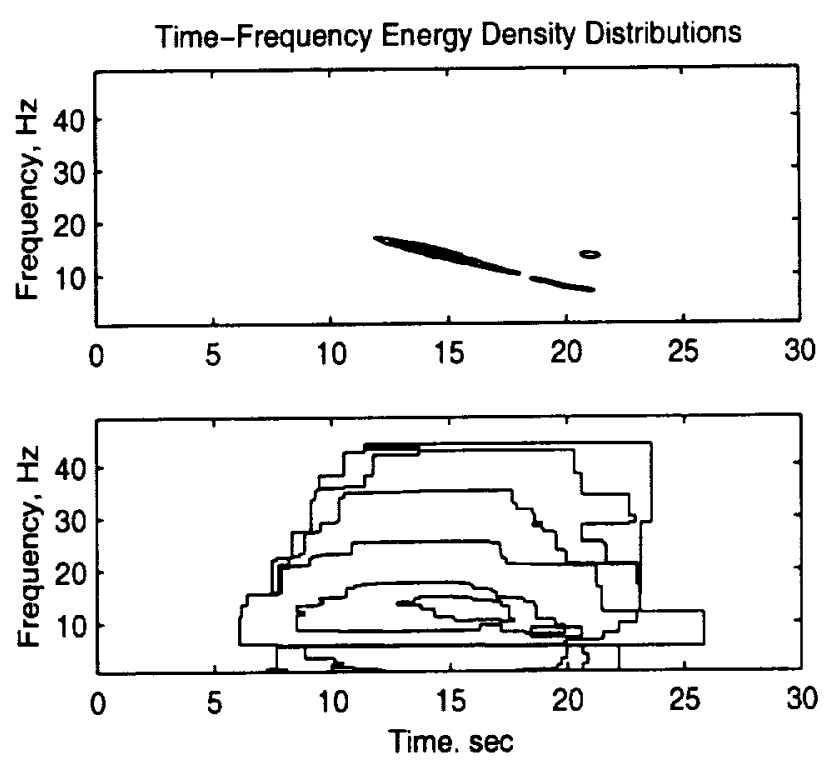

Figure 25: Contour plots from original wavelet decomposition (top) and SVD-filtered (bottom, log-scale) scalograms of F18-SRA wingtip accelerometer response.

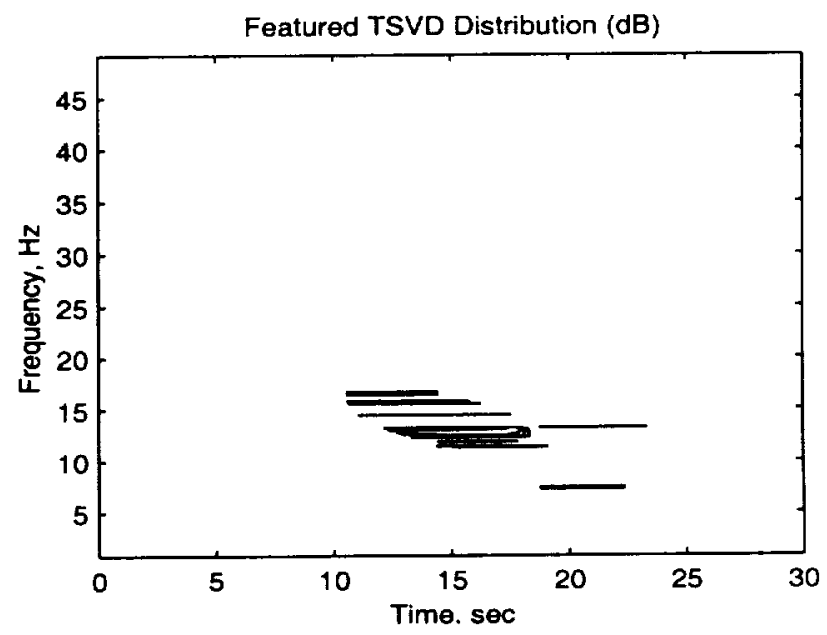

Figure 26: Contour plot of TSVD-filtered scalogram of F18-SRA multisine wingtip accelerometer response.

SVD filter (bottom plot) attempts to bound the entire time-frequency response, as in the other cases, whereas the TSVD-filtered output in figure 26 distinguishes fine frequency divisions over short time intervals. The $13 \mathrm{~Hz}$ and $7 \mathrm{~Hz}$ lines near $20 \mathrm{sec}$ are very distinct and precisely tuned to the specific frequencies.

A TSVD-filtered reconstruction of the original wingtip response is in figure 27 , which corresponds to the contour plot of figure 26. Both the SVD (bottom contour plot, figure 25) and TSVD contour and time history plots show dominance from the $12-13 \mathrm{~Hz}$ mode just around $15 \mathrm{sec}$. Since the control system is active, this mode corresponds to the stabilator mode which is excited by the aileron multisine in closed loop. 


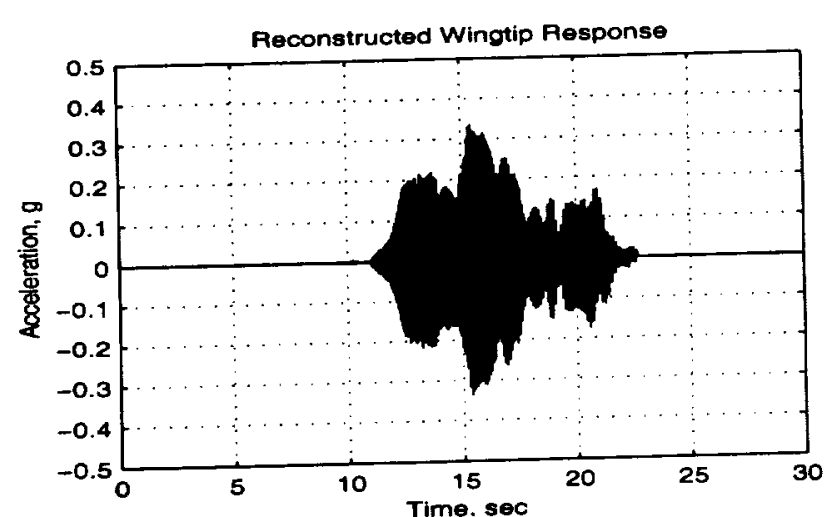

Figure 27: Reconstructed TSVD-filtered F18-SRA wingtip accelerometer response from multisine aileron input.
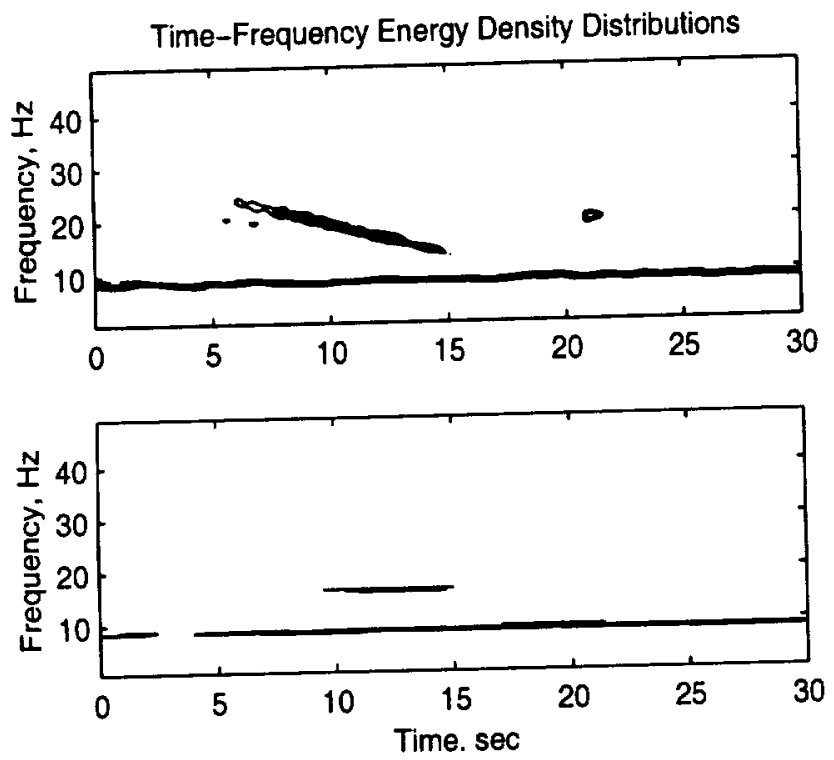

Figure 28: Contour plots from original wavelet decomposition (top) and TSVD-filtered (bottom, log-scale), scalograms of F18-SRA fuselage lateral accelerometer.

In a final example, figure 28 shows a persistent oscillation of the wing bending mode at $8-9 \mathrm{~Hz}$ (first fuselage and wing bending modes) from the fuselage lateral accelerometer for the same multisine aileron input. The top plot is the original energy density, and the bottom is the log-scaled TSVD-filtered distribution. The SVD distribution is similar to that in figure 25 , so is not shown. The TSVD defines the persistent mode very well while also detecting the dominant part of the chirp near $16 \mathrm{~Hz}$ $(10-15 \mathrm{sec})$. The final part of the energy density distribution after $20 \mathrm{sec}$ at $20 \mathrm{~Hz}$, however, is not detected since it is relatively less significant than the $8-9 \mathrm{~Hz}$ modes. Time-dependent modal frequency and damping ratios estimated with the TSVD filter are displayed in figure 29 to show the relatively low damping of the steady oscillation at $8-9 \mathrm{~Hz}$ compared to modes at 19,17 , and $15 \mathrm{~Hz}(10-15$ sec) corresponding to fuselage second bending, wing second bending, and wing first torsion modes, respectively.
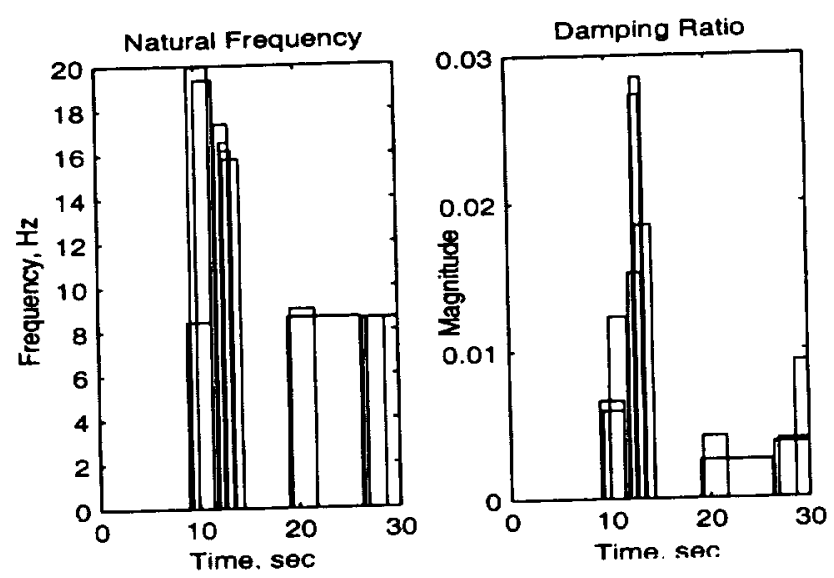

Figure 29: TSVD estimated modal frequencies (left) and damping ratios (right) from F18-SRA fuselage accelerometer.

\section{Conclusions}

Nonstationary dynamics data analysis is discussed from the perspective of time-frequency and multiscale resolution analyses. Wavelet decomposition of dynamics data using a discrete harmonic basis constructed from the Morlet wavelet establishes a time-frequency representation for modal parameter estimation and system identification procedures. Automated procedures to discern and extract regions of the time-frequency energy density distribution that are significant for dynamics analysis are presented. The methods are derived from the singular value decomposition (SVD) of a time-frequency distribution.

Examples of simulated sinusoids, a testbed aeroelastic system, aeroelastic flight data from a flutter experiment, and aeroservoelastic data from an F18 are used to demonstrate and compare the two SVD filtering methods. One method using a standard SVD demonstrates the ability to discriminate the most significant and other less dominant dynamics. Its performance may depend on a time-frequency window determined by the input. Regions outside of the input time-frequency path relate to information about noise, unmodeled dynamics, and nonlinearity.

Another method called the transformed-SVD, or TSVD, optimally rotates the singular vectors to create a more precise filter for finer tracking, but may ignore the less significant dynamics for the same order filter as the standard SVD. Both methods complement each other and show promise for automatic feature extraction supplemented with noise removal, residual analysis, and uncertainty estimation. These filtering methods contribute to nonstationary dynamics data analysis at multiple scales in the time-frequency framework. 


\section{$\underline{\text { Appendix }}$}

Given a general harmonic signal

$$
x(t)=k(t) \cos (\phi(t) t)
$$

the WT of $x(t)$ is

$$
W(a, \tau)=\sqrt{a} k(t) e^{-\left(a \phi(t)-\omega_{0}\right)^{2}} e^{i \phi(t) \tau} .
$$

For fixed dilation parameter $a_{i}$ (equivalently fixed frequency $\omega)$, the modulus and phase of the WT of $x(t)$ are

$$
\begin{aligned}
\left|W\left(a_{i}, \tau\right)\right| & =\sqrt{a_{i}} k(t) e^{-\left(a_{i} \phi(t)-\omega_{0}\right)^{2}} \\
\left.\angle W\left(a_{i}, \tau\right)\right] & =\phi(t) \tau
\end{aligned}
$$

Instantaneous frequency of a signal in this case can be expressed as ${ }^{[36]}$

$$
\phi(t)=\frac{1}{2 \pi} \frac{d}{d t}\left(\angle\left[W\left(a_{i}, \tau\right)\right]\right)
$$

This shows that a general time-varying envelope $k(t)$ or phase $\phi(t)$ of the signal can be determined from the modulus and phase of the WT for each fixed wavelet frequency.

More specifically, from the impulse response of a single degree-of-freedom viscous damper

$$
x(t)=A e^{-\zeta \omega_{n} t} \cos \left(\omega_{d} t+\phi_{o}\right)
$$

we have from equation 5 ,

$$
\begin{aligned}
& k(t)=\frac{\left|W\left(a_{i}, \tau\right)\right|}{\sqrt{a_{i} e^{-\left(a_{i} \phi(t)-\omega_{0}\right)^{2}}}}=A e^{-\zeta \omega_{n} t} \\
& \phi(t) t=\left\langle\left[W\left(a_{i}, \tau\right)\right]=\omega_{d} t+\phi_{o}\right.
\end{aligned}
$$

For a constant wavelet frequency line corresponding to $a_{i}$ over time $\tau$ in the $(a, \tau)$ plane, estimation of the WT linear phase variation (or mean value of the instantaneous frequency over time, from eq. 6) gives $\phi(t) \approx \omega_{d}$, and the envelope decay rate is $\zeta \omega_{n}$. Natural frequency $\omega_{n}$ and modal damping ratio $\zeta$ are therefore derived. The WT becomes a complex representation of the original real signal from which the signal eigenvalues are computed without any approximation of their range.

Multiple degree-of-freedom systems are analyzed similarly by noting that the dilated Morlet wavelet is a bandpass filter. With sufficient resolution of dilation $a_{i}$, damped modal frequencies $\omega_{d_{i}}=\frac{\omega_{0}}{a_{i}}$ can be discriminated. To recap, the decay rate of the envelope of each mode is calculated from the log-slope of the wavelet modulus decay, and damped modal frequency is estimated as the linear phase variation of the WT as a function of time. Adequate frequency resolution can be enforced with the multiscaled compact harmonic Morlet wavelets.

\section{References}

1. Berry, Michael W., Z. Drmač, and E.R. Jessup, "Matrices, Vector Spaces, and Information Retrieval", SIAM Review, 41(2), pp. 335-362.

2. Brenner, M.J. and E. Feron, "Wavelet Analysis of F/A-18 Aeroelastic and Aeroservoelastic Flight Test Data", AIAA Structures, Structural Dynamics, and Materials Conference, Kissimmee, FL, Apr 1997, AIAA-97-1216, NASA-TM-4793.

3. Brenner, Marty and R. Lind, "Wavelet-Processed Flight Data for Robust Aeroservoelastic Stability Margins", AIAA Journal of Guidance, Control, and Dynamics, 21(6), Nov-Dec 1998, pp. 823-829.

4. Chapa, Joseph O. and R.M. Rao, "Algorithms for Designing Wavelets to Match a Specified Signal", IEEE Transactions on Signal Processing, 48(12), Dec 2000, pp. 3395-3406.

5. Cohen, Leon, Time-Frequency Analysis, PrenticeHall, Englewood Cliffs, NJ, 1995.

6. Davidson, Keith L. and P.L. Loughlin, "Instantaneous spectral moments", Journal of the Franklin Institute, 337, 2000, pp. 421-436.

7. De Cock, Katrien, and B. De Moor, "Subspace angles between linear stochastic models", IEEE Conference on Decision and Control, Sydney, Australia, Dec 2000.

8. Donoho, D.L., M. Vetterli, R.A. DeVore, and I. Daubechies, "Data Compression and Harmonic Analysis", IEEE Transactions on Information Theory, 44(6), Oct 1998, pp. 2435-2476.

9. Erbay, Hasan and J.L. Barlow, "Recursive ULV Decomposition", SPIE Proceedings of Advanced Signal Processing Algorithms, Architectures, and Implementations $X, \mathbf{4 1 1 6}$, San Diego, CA, Jul 2000, pp. 157-166.

10. Feron, Eric, M. Brenner, J. Paduano, and A. Turevskiy, "Time-Frequency Analysis for Transfer Function Estimation and Application to the F18SRA", AIAA Journal of Guidance, Control, and Dynamics, 21(2), Mar-Apr 1998, pp. 375-382.

11. Francos, A. and M. Porat, "Analysis and Synthesis of Multicomponent Signals using Positive TimeFrequency Distributions", IEEE Transactions on Signal Processing, 47(2), Feb 1999, pp. 493-504.

12. Gilliatt, H.C., T.W. Strganac, and A.J. Kurdila, "Nonlinear Aeroelastic Response of an Airfoil", AIAA Aerospace Sciences Meeting, Reno, NV, Jan 1997, AIAA-97-0459. 
13. Gilyard, Glenn B. and J.W. Edwards, Real-time Flutter Analysis of an Active Flutter-Suppression System on a Remotely Piloted Research Aircraft, NASA TM-84901, Jan 1983.

14. Groutage, Dale and D. Bennink, "Feature Sets for Nonstationary Signals Derived from Moments of the Singular Value Decomposition of CohenPosch (Positive-Time-Frequency) Distributions" IEEE Transactions on Signal Processing, 48(5), May 2000, pp. 1498-1503.

15. Groutage, D. and D. Bennink, "A New Matrix Decomposition Based on Transforming the Basis Sets of the Singular Value Decomposition Yields Principal Features for Time-Frequency Distributions", SPIE Proceedings of Advanced Signal Processing Algorithms, Architectures, and Implementations $X$, 4116, San Diego, CA, Jul 2000, pp. 66-79.

16. Groutage, Dale, "A Fast Algorithm for Computing Minimum Cross-Entropy Positive Time-Frequency Distributions", IEEE Transactions on Signal Processing, 45(8), Aug 2000, pp. 1954-1970.

17. Hlawatsch, Franz, G. Matz, H. Kirchauer, and W. Kozek, "Time-Frequency Formulation, Design, and Implementation of Time-varying Optimal Filters for Signal Estimation", IEEE Transactions on Signal Processing, 48(5), May 2000, pp. 1417-1432.

18. Hubbard, Barbara B., The World According to Wavelets, A.K. Peters, Wellesley MA, 1998.

19. Iyama, H. and J. Kuwamura "Application of wavelets to analysis and simulation of earthquake motions", Earthquake Engineering and Structural Dynamics, 28(3), 1999, pp. 255-272.

20. Jiang, E.P. and M. Berry, "Solving total least squares problems in information retrieval", Linear Algebra and its Applications, 316, 2000, pp. 137156.

21. Ko, J., A. Kurdila and T. Strganac, "Nonlinear Control Theory for a Class of Structural Nonlinearities in a Prototypical Wing Section", AIAA Aerospace Sciences Meeting, Reno NV, Jan 1997, AIAA-97-0580.

22. Krim, Hamid, D. Tucker, and S. Mallat, "On Denoising and Best Signal Representation", IEEE Transactions on Information Theory, 45(7), Nov 1999, pp. 2225-2338.

23. Le, Dzu K., "Application of Sampling Theorems in Wavelet Spaces to Multiresolution Visualization and Data Segmentation", SPIE Proceedings of Wavelet Applications in Signal and Image Processing III, 2569, Bellingham, WA, Jul 1995, pp. 220233.
24. Le, Dzu K., A.K. Owen, and D.L. Mattern, "Multiscale Analysis of Stall Inception and Instabilities in an Axi-Centrifugal Turboshaft Engine", AIAA Joint Propulsion Conference, Lake Buena Vista, FL, Jul 1996, AIAA-96-3174.

25. Lind, Rick, K. Snyder, and M. Brenner, "Investigating Transient and Limit Cycle Behaviors of a Nonlinear Structure by Wavelet Transforms", AIA A Structures, Structural Dynamics, and $\mathrm{Ma}$ terials Conference, Apr 1998, AIAA-98-1808.

26. Liu, B. and S.-F. Ling, "On the Selection of Informative Wavelets for Machinery Diagnosis", Mechanical Systems and Signal Processing, 13(1), 1999, pp. 145-162.

27. Loughlin, P., F. Cakrak, and L. Cohen, "Conditional Moments Analysis of Transients with Application to Helicopter Fault Data", Mechanical Systems and Signal Processing, 14(4), 1999, pp. 511522 .

28. Mallat, Stéphane, A Wavelet Tour of Signal Processing, Academic Press, 1999.

29. Marinovic, N.M., W. A. Smith, R.C. Waag, and K.J. Parker, "Limits Imposed by Tissue on SVDWigner Filtering to Obtain the Intrinsic Frequency Variation of Ultrasonic Echo Waveforms", IEEE Ultrasonics Symposium, 2, 1989, pp. 945-950.

30. Mastroddi, F. and A. Bettoli, "Nonlinear Aeroelastic System Identification via Wavelet Analysis in the Neighborhood of a Limit Cycle", CEAS International Forum on Aeroelasticity and Structural Dynamics, Williamsburg, VA, NASA-CP1999-209136/PT2, pp. 857-866.

31. McClure, Mark R. and L. Carin, "Matching Pursuits with a Wave-Based Dictionary", IEEE Transactions on Signal Processing, 45(12), Dec 1997, pp. 2912-2927.

32. Nelson, Douglas J. and W. Wysocki, "Cross Spectral Methods with an Application to Speech Processing", SPIE Proceedings of Advanced Signal Processing Algorithms, Architectures, and Implementations IX, 3807, Denver, CO, Jul 1999, pp. 552-563.

33. Nelson, Douglas J., et. al., "Denoising Using TimeFrequency and Image Processing Methods", SPIE Proceedings of Advanced Signal Processing Algorithms, Architectures, and Implementations IX, 3807, Denver, CO, Jul 1999, pp. 564-581.

34. Olmo, G., F. Dovis, P. Benotto, C. Calosso, and P. Passaro, "Instrument-Independent Analysis of Music by Means of the Continuous Wavelet Transform", SPIE Proceedings of Wavelet Applications 
in Signal and Image Processing VII, 3813, Denver, CO, Jul 1999, pp. 716-726.

35. Pendleton, E., D. Bessette, P. Field, G. Miller, and K. Griffin, "Active Aeroelastic Wing Flight Research Program: Technical Program and Model Analytical Development", AIAA Journal of Aircraft, 37(4), Jul-Aug 2000, pp. 554-561.

36. Ruzzene, M., A. Fasana, L. Garibaldi, and B. Piombo, "Natural Frequencies and Dampings Identification using Wavelet Transform: Application to Real Data", Mechanical Systems and Signal Processing, 11(2), 1997, pp. 207-218.

37. Shah, S.I., A. El-Jaroudi, P.J. Loughlin, L.F. Chaparro, "Signal synthesis and positive time frequency distributions", Journal of the Franklin Institute, 337, 2000, pp. 317-328.

38. Shensa, Mark J., "Discrete Inverses for Nonorthogonal Wavelet Transforms", IEEE Transactions on Signal Processing, 44(4), Apr 1996, pp. 798-807.

39. Shin, K., J.K. Hammond, and P.R. White, "Iterative SVD Method for Noise Reduction of Low-Dimensional Chaotic Time Series", Mechanical Systems and Signal Processing, 13(1), 1999, pp. 115-124.

40. Staszewski, W.J., "Structural and Mechanical Damage Detection Using Wavelets", Shock and Vibration Digest, 30(6), Nov 1998, pp. 457-472.

41. Sun, Mingui, M.L. Scheuer, R.J. Sclabassi, "Decomposition of biomedical signals for enhancement of their time-frequency distributions", Journal of the Franklin Institute, 337, 2000, pp. 453-467.

42. Taswell, Carl, "Top-Down and Bottom-Up Tree Search Algorithms for Selecting Bases in Wavelet Packet Transforms", Wavelets and Statistics, Lecture Notes in Statistics, Springer-Verlag, 1995.

43. Venkatachalam, Vidya and J.L. Aravena, "Nonstationary Signal Classification Using Pseudo Power Signatures: The Matrix SVD Approach", IEEE Transactions on Circuits and Systems - II 46(12), Dec 1999, pp. 1497-1505.

44. Vetterli, Martin and Jelena Kovačević, Wavelets and Subband Coding, Prentice-Hall PTR, Upper Saddle River, NJ, 1995.

45. Wong, L.A., J.C. Chen, "Nonlinear and chaotic behavior of structural system investigated by wavelet transform techniques", International Journal of Nonl-Linear Mechanics, 36, 2001, pp. 221-235. 
Pubic Public reporting burden for this collection of intormation is estmated loction of intormation Send comments regarding this burden estimate or any other aspect of this collection 1204 . Arlington, maintaining the data needed, and completing and revisingon Headquarters Services. Directorate for intormation Operations and Repd

\begin{tabular}{l|l} 
VA 22202-4302, and to the Otice of Management and Budget, Papenwik Reduch & 2. REPORT DATE \\
\hline 1. AGENCY USE ONLY (Leave blank)
\end{tabular}

\begin{tabular}{|l|r}
\hline 1. AGENCY USE ONLY (Leave blank) & $\begin{array}{r}\text { 2. REPORT DATE } \\
\text { April 200I }\end{array}$ \\
\hline
\end{tabular}

3. REPOAT TYPE AND DATES COVERED

Technical Memorandum

4. TITLE AND SUBTITLE

Nonstationary Dynamics Data Analysis With Wavelet-SVD Filtering

WU 529-50-04-00-RR-00-000

6. AUTHOR(S)

Marty Brenner and Dale Groutage

7. PERFORMING ORGANIZATION NAME(S) ANO ADDRESS(ES)

NASA Dryden Flight Research Center

P.O. Box 273

Edwards, California 93523-0273

9. SPONSORING/MONITORING AGENCY NAME(S) AND ADDRESS(ES)

AGENCY REPORT NUMBER

National Aeronautics and Space Administration

NASAVTM-2001-210391

Washington, DC 20546-0001

11. SUPPLEMENTARY NOTES

Modified from paper presented at 42nd AIAA/ASME/ASCE/AHS/ASC Structures, Structural Dynamics, and Materials Conference, Seattle, Washington, April 16-19, 2001. Dale Groutage, Naval Surface Warfare Center (NSWC), Bremerton, Washington.

12a. DISTRIBUTIONAVALABILITY STATEMENT

12b. DISTRIBUTION CODE

Unclassified--Unlimited

Subject Category 66

This report is available at http://www.dfrc.nasa.gov/DTRS/

13. ABSTRACT (Maximum 200 words)

Nonstationary time-frequency analysis is used for identification and classification of aeroelastic and aeroservoelastic dynamics. Time-frequency multiscale wavelet processing generates discrete energy density distributions. The distributions are processed using the singular value decomposition (SVD). Discrete density functions derived from the SVD generate moments that detect the principal features in the data. The SVD standard basis vectors are applied and then compared with a transformed-SVD, or TSVD, which reduces the number of features into more compact energy density concentrations. Finally, from the feature extraction, wavelet-based modal parameter estimation is applied.

\begin{tabular}{|c|c|c|c|}
\hline \multirow{2}{*}{\multicolumn{3}{|c|}{$\begin{array}{l}\text { 14. SUBJECT TERMS } \\
\text { Aeroelasticity, Aeroservoelasticity, Singular value decomposition, System } \\
\text { identification, Time-frequency analysis, Wavelet analysis }\end{array}$}} & \multirow{2}{*}{\begin{tabular}{|l|} 
15. NUMBER OF PAGES \\
22 \\
16. PRICE CODE
\end{tabular}} \\
\hline & & & \\
\hline $\begin{array}{l}\text { 17. SECURITY CLASSIFICATION } \\
\text { OF REPORT } \\
\text { Unclassified }\end{array}$ & $\begin{array}{l}\text { 18. SECUAITY CLASSIFICATION } \\
\text { OF THIS PAGE } \\
\text { Unclassified }\end{array}$ & $\begin{array}{l}\text { 19. SECURITY CLASSIFICATION } \\
\text { OF ABSTRACT } \\
\text { Unclassified }\end{array}$ & $\begin{array}{l}\text { 20. LIMITATION OF ABSTRACT } \\
\text { Unlimited }\end{array}$ \\
\hline & & & $\begin{array}{l}\text { Indard Form } 298 \text { (Rev. 2-89) } \\
\text { ceitod by ANSI Std Z39-18 } \\
102\end{array}$ \\
\hline
\end{tabular}


National Aeronautics and

Space Administration

Code JTT

Washington, D.C. 20546-0001

SPECIAL FOURTH-CLASS RATE POSTAGE AND FEES PAID

NASA

PERMIT No G27

USA

Official Business

Penalty for Private Use, $\$ 300$

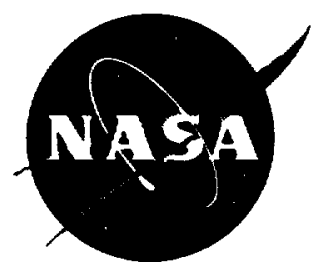

POSTMASTER: If Undeliverable (Section 158

Postal manual) Do Not Retum 